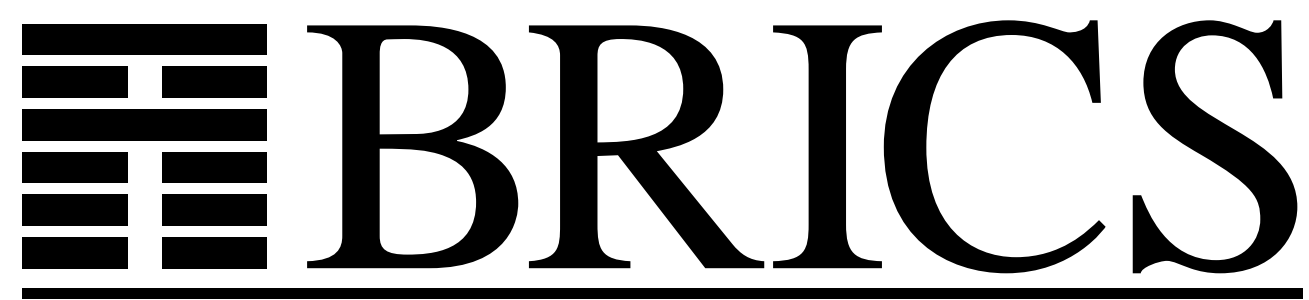

Basic Research in Computer Science

\title{
On the Arithmetical Content of Restricted Forms of Comprehension, Choice and General Uniform Boundedness
}

Ulrich Kohlenbach

RS-97-23 
Copyright (c) 1997, $\quad$ BRICS, Department of Computer Science University of Aarhus. All rights reserved.

Reproduction of all or part of this work is permitted for educational or research use on condition that this copyright notice is included in any copy.

See back inner page for a list of recent BRICS Report Series publications. Copies may be obtained by contacting:

\author{
BRICS \\ Department of Computer Science \\ University of Aarhus \\ Ny Munkegade, building 540 \\ DK-8000 Aarhus C \\ Denmark \\ Telephone: +4589423360 \\ Telefax: $\quad+4589423255$ \\ Internet: BRICS@brics.dk
}

BRICS publications are in general accessible through the World Wide Web and anonymous FTP through these URLs:

http://www.brics.dk

ftp: //ftp.brics.dk

This document in subdirectory RS / 97/23/ 


\title{
On the arithmetical content of restricted forms of comprehension, choice and general uniform boundedness
}

\author{
Ulrich Kohlenbach \\ BRICS* $^{*}$ \\ Department of Computer Science \\ University of Aarhus \\ Ny Munkegade, Bldg. 540 \\ DK-8000 Aarhus C, Denmark \\ kohlenb@brics.dk
}

August 5, 1997

\begin{abstract}
In this paper the numerical strength of fragments of arithmetical comprehension, choice and general uniform boundedness is studied systematically. These principles are investigated relative to base systems $\mathcal{T}_{n}^{\omega}$ in all finite types which are suited to formalize substantial parts of analysis but nevertheless have provably recursive function(al)s of low growth. We reduce the use of instances of these principles in $\mathcal{T}_{n}^{\omega}$-proofs of a large class of formulas to the use of instances of certain arithmetical principles thereby determining faithfully the arithmetical content of the former. This is achieved using the method of elimination of Skolem functions for monotone formulas which was introduced by the author in a previous paper.

As corollaries we obtain new conservation results for fragments of analysis over fragments of arithmetic which strengthen known purely first-order conservation results.
\end{abstract}

*Basic Research in Computer Science, Centre of the Danish National Research Foundation. 


\section{Introduction}

This paper studies the numerical strength of fragments $\Gamma$ of arithmetical comprehension, choice and uniform boundedness relative to weak base systems, formulated in the language of all finite types, which are suited to formalize substantial parts of analysis.

In a previous paper ([12]) we have introduced a hierarchy $\mathrm{G}_{n} \mathrm{~A}^{\omega}$ of systems where the definable functions correspond to the well-known Grzegorczyk hierarchy. These systems extended by the schema of full quantifier-free choice

$$
\begin{aligned}
& A C^{\rho, \tau} \text {-qf }: \forall x^{\rho} \exists y^{\tau} A_{0}(x, y) \rightarrow \exists Y^{\tau(\rho)} \forall x^{\rho} A_{0}(x, Y x), \\
& A C \text {-qf }:=\bigcup_{\rho, \tau \in \mathbf{T}}\left\{A C^{\rho, \tau} \text {-qf }\right\},
\end{aligned}
$$

where $A_{0}$ is a quantifier-free formula, ${ }^{1}$ and various non-constructive analytical axioms $\Delta$, having the form

$$
\forall x^{\rho} \exists y \leq_{\tau} s x \forall z^{\delta} A_{0}(x, y, z)
$$

including a generalized version of the binary König's lemma, allow to carry out a great deal of classical analysis even for $n=2,3$. The axioms $\Delta$ and AC-qf do not contribute to the growth of extractable uniform bounds which in the particular case of $\mathrm{G}_{2} \mathrm{~A}^{\omega}$ are polynomials (see [12],[14] and in particular [10] for more information).

In contrast to this, fragments of arithmetical comprehension and choice as well as generalizations of our principle of uniform $\Sigma_{1}^{0}$-boundedness (from [12]) to more complex formulas do contribute significantly to the arithmetic strength of the base systems. In [13] we developed a general method to calibrate faithfully this contribution and applied it to instances of $\Pi_{1}^{0}$ comprehension and $\Pi_{1}^{0}$-choice. These results were then used in [15] to determine the arithmetical strength of single sequences of instances of the BolzanoWeierstraß theorem for bounded sequences in $\mathbb{R}^{d}$, the Ascoli-lemma and others.

In this paper we give a systematic treatment of the whole arithmetical hierarchy for comprehension, choice and uniform boundedness and determine precisely their arithmetical strength. We also consider much more complex formulas to be proved in these systems than we did in our previous papers.

\footnotetext{
${ }^{1}$ Throughout this paper $A_{0}, B_{0}, C_{0}, \ldots$ denote quantifier-free formulas. We allow bounded number quantifiers $\forall x \leq_{0} t, \exists x \leq_{0} t$ to occur in $A_{0}, B_{0}, C_{0}, \ldots$ since they can be expressed in a quantifier-free way using the bounded search-functional $\mu_{b}$ from $\mathrm{G}_{n} \mathrm{~A}^{\omega}$. T denotes the set of all finite types.
} 
In the following let us discuss now some of the difficulties one has to deal with in order to achieve this goal and which indicate already the type of results one can expect. For simplicity we restrict ourselves for the moment to the second-order system $\mathrm{EA}^{2}+\mathrm{AC}^{0,0}$-qf instead of $\mathrm{G}_{n} \mathrm{~A}^{\omega}+\mathrm{AC}$-qf $+\Delta$ (which we actually are going to consider below).

$\mathrm{EA}^{2}$ is an extension of Kalmar-elementary arithmetic (with number quantifiers) EA obtained by adding $n$-ary function quantifiers (for every $n \geq 1)^{2}$ and the schema of explicit definition of functions

$$
E D: \exists f \forall \underline{x}(f(\underline{x})=t[\underline{x}]),
$$

where $t$ is a number term of $\mathrm{EA}^{2}$ and $\underline{x}$ is a tuple of number variables. Furthermore $\mathrm{EA}^{2}$ contains the schema of quantifier-free induction for all quantifier-free formulas of $\mathrm{EA}^{2}$ which may contain function parameters. Finally $\mathrm{EA}^{2}$ contains constants and their defining equations for all elementary recursive functionals of type-level 2 .

In $\mathrm{EA}^{2}$ the schema of quantifier-free induction can be expressed equivalently as a single axiom

$$
\text { QF-IA : } \forall f\left(f(0)=0 \wedge \forall x\left(f(x)=0 \rightarrow f\left(x^{\prime}\right)=0\right) \rightarrow \forall x(f(x)=0)\right) \text {. }
$$

Let us consider furthermore the restriction of arithmetical choice to $\Pi_{1}^{0}$ - (or equivalently to $\left.\Sigma_{2^{-}}^{0}\right)$ formulas of $\mathcal{L}\left(\mathrm{EA}^{2}\right)$ which like QF-IA can be expressed as a single second-order axiom $\forall f \Pi_{1}^{0}$-AC $(f)$, where ${ }^{3}$

$$
\Pi_{1}^{0}-\mathrm{AC}(f): \equiv \forall a^{0}\left(\forall x^{0} \exists y^{0} \forall z^{0}(f(a, x, y, z)=0) \rightarrow \exists g \forall x, z(f(a, x, g x, z)=0)\right) .
$$

Now by iteration one easily verifies that $\mathrm{EA}^{2}+\forall f \Pi_{1}^{0}$-AC $(f)$ proves already full arithmetical choice. So in order to prevent the arithmetical hierarchy of choice principles from collapsing we restrict ourselves to single instances of $\forall f \Pi_{1}^{0}$-AC $(f)$ which later on are allowed however to depend on the parameters of the theorem to be proved. For the moment we forbid completely the occurrence of function parameters in $\Pi_{1}^{0}$-AC, i.e. we consider the schema

$$
\Pi_{1}^{0} \mathrm{AC}^{-}: \forall x^{0} \exists y^{0} A(x, y) \rightarrow \exists g \forall x A(x, g x),
$$

where $A(x, y)$ is a $\Pi_{1}^{0}$-formula without function parameters.

\footnotetext{
${ }^{2}$ Since coding of finite tuples of numbers is available in EA one can in fact restrict oneself to unary function variables.

${ }^{3}$ The universal closure with respect to number parameters $a^{0}$ is superfluous for $\forall f \Pi_{1}^{0}$ $\mathrm{AC}(f)$ since it can be captured by the universal closure $\forall f$. However below we consider single instances $\Pi_{1}^{0}$ - $\mathrm{AC}(\xi)$ of $\forall f \Pi_{1}^{0}$-AC $(f)$ where it does make a difference.
} 
As a starting point for the introduction into our general program let us consider now the following question:

What arithmetical statements are provable in $\mathrm{EA}^{2}+\mathrm{AC}^{0,0}-\mathrm{qf}+\Pi_{1}^{0}-\mathrm{AC}^{-}$?

A first observation is that $\Pi_{1}^{0}-\mathrm{AC}^{-}$proves $\Pi_{1}^{0}-\mathrm{CA}^{-}$, i.e.

$$
\exists f \forall x(f(x)=0 \leftrightarrow A(x)),
$$

where $A(x)$ is a $\Pi_{1}^{0}$-formula without function parameters. Combined with the axiom QF-IA this yields every function parameter-free instance of $\Sigma_{1}^{0}$ IA. Hence the first-order system EA $+\Sigma_{1}^{0}$-IA is a subsystem of $\mathrm{EA}^{2}+\mathrm{AC}^{0,0}$-qf $+\Pi_{1}^{0}-\mathrm{AC}^{-}$.

What is the precise relationship between $\mathrm{EA}^{2}+\mathrm{AC}^{0,0}-\mathrm{qf}+\Pi_{1}^{0}-\mathrm{AC}^{-}$and $\mathrm{EA}$ $+\Sigma_{1}^{0}$-IA?

It will turn out that the former theory is conservative over the latter for some formulas, including $\Pi_{3}^{0}$-sentences, but not for all formulas.

That $\mathrm{EA}^{2}+\mathrm{AC}^{0,0}$-qf cannot be conservative over $\mathrm{EA}+\Sigma_{1}^{0}$-IA without some restriction imposed on the formulas follows from the following observation:

By applying the functional $\Phi_{\max } f x:=\max _{i \leq x}(f(i))$ to the function $g$ in $\Pi_{1}^{0}$ - $\mathrm{AC}^{-}$ one obtains the corresponding instance of the so-called (bounded) collection principle for $\Pi_{1}^{0}$-formulas

$$
\Pi_{1}^{0}-\mathrm{CP}: \forall x \leq a \exists y A(x, y) \rightarrow \exists z \forall x \leq a \exists y \leq z A(x, y),
$$

where $A \in \Pi_{1}^{0}$.

So $\mathrm{EA}^{2}+\mathrm{AC}^{0,0}{ }_{\text {-qf }}+\Pi_{1}^{0}-\mathrm{AC}^{-}$proves every function parameter-free instance of $\Pi_{1}^{0}$-CP, i.e. $\mathrm{EA}+\Pi_{1}^{0}$ - $\mathrm{CP}$ is a subsystem of $\mathrm{EA}^{2}+\mathrm{AC}^{0,0}-\mathrm{qf}+\Pi_{1}^{0}-\mathrm{AC}^{-}$.

It is well-known (see [18]) that there exists an instance $A$ of $\Pi_{1}^{0}$-CP which is not provable in $\mathrm{EA}+\Sigma_{1}^{0}$-IA. On the other hand $\mathrm{EA}+\Pi_{1}^{0}$ - $\mathrm{CP}$ is $\Pi_{3}^{0}$ conservative over EA $+\Sigma_{1}^{0}$-IA by a result due to $\mathrm{H}$. Friedman and (implicitly) J.Paris/L.Kirby [17] (see e.g. [7] for details). The universal closure of the instance $A$ of $\Pi_{1}^{0}$-CP can be shown to be equivalent to a $\Pi_{4}^{0}$-sentence in EA $+\Sigma_{1}^{0}$-IA. Hence $\mathrm{EA}^{2}+\mathrm{AC}^{0,0}$-qf $+\Pi_{1}^{0}-\mathrm{AC}^{-}$is not $\Pi_{4}^{0}$-conservative over $\mathrm{EA}$ $+\Sigma_{1}^{0}$-IA.

Here is another arithmetical use of $\Pi_{1}^{0}-\mathrm{AC}^{-}$we can make relative to $\mathrm{EA}^{2}+$ $\mathrm{AC}^{0,0}{ }_{\text {-qf: }}$ 
As mentioned above, $\Pi_{1}^{0}-\mathrm{CA}^{-}$is a trivial consequence of $\Pi_{1}^{0}-\mathrm{AC}^{-}$(in the presence of classical logic). Now combining $\Pi_{1}^{0}-\mathrm{CA}^{-}$with $\mathrm{AC}^{0,0}{ }_{\text {-qf }}$ one can easily prove $\Delta_{2}^{0}-\mathrm{CA}^{-}$and therefore every function parameter-free instance of $\Delta_{2}^{0}$-IA. Hence EA $+\Delta_{2}^{0}$-IA is a subsystem of $\mathrm{EA}^{2}+\mathrm{AC}^{0,0}-\mathrm{qf}+\Pi_{1}^{0}-\mathrm{AC}^{-}$as well even if the functional $\Phi_{\max }$ were not included in $\mathrm{EA}^{2}$.

So the arithmetical strength of $\Pi_{1}^{0}$ - $\mathrm{AC}^{-}$depends heavily on the second-order axioms, like $\mathrm{QF}-\mathrm{IA}, \mathrm{AC}^{0,0}$-qf and the characterizing axioms for functionals as $\Phi_{\max }$, which are available in the context in which $\Pi_{1}^{0}-\mathrm{AC}^{-}$is considered. ${ }^{4}$

As a special corollary of the results of this paper it follows that $\mathrm{EA}^{2}+\mathrm{AC}^{0,0}$-qf $+\Pi_{k}^{0}-\mathrm{AC}^{-}$is $\Pi_{k+2}^{0}$-conservative over $\mathrm{EA}+\Sigma_{k}^{0}$-IA, which implies the result of H. Friedman, J.Paris/L.Kirby. Furthermore we show that $\mathrm{EA}^{2}+\mathrm{AC}^{0,0}-\mathrm{qf}+\Pi_{k}^{0}-\mathrm{AC}^{-}$is conservative over $\mathrm{EA}+\Sigma_{k}^{0}$-IA w.r.t. monotone formulas of arbitrary complexity. These results are sensitive to small changes of the base system $\mathrm{EA}^{2}$ : E.g. if we add the primitive recursive functional $\Phi_{i t}$ defined by

$$
\Phi_{i t} f g 0:=g(0) \quad \Phi_{i t} f g x^{\prime}:=f\left(x, \Phi_{i t} f g x\right)
$$

to $\mathrm{EA}^{2}$, then the Ackermann-function becomes provably total in $\mathrm{EA}^{2}+\Phi_{i t}+\mathrm{AC}^{0,0}$-qf $+\Pi_{1}^{0}-\mathrm{AC}^{-}$and the resulting system proves the consistency of $\mathrm{EA}+\Sigma_{1}^{0}$-IA: $\mathrm{EA}^{2}+\Phi_{i t}+\mathrm{AC}^{0,0}$-qf proves the second-order axiom of $\Sigma_{1}^{0}$-induction. Combined with $\Pi_{1}^{0}-\mathrm{CA}^{-}$one obtains every function parameterfree instance of $\Sigma_{2}^{0}$-IA. Hence EA $+\Sigma_{2}^{0}$-IA (which is known to prove the totality of the Ackermann-function as well as the consistency of EA $+\Sigma_{1}^{0}$-IA) is a subsystem of $\mathrm{EA}^{2}+\Phi_{i t}+\mathrm{AC}^{0,0}-\mathrm{qf}+\Pi_{1}^{0}-\mathrm{AC}^{-}$.

Using a more involved argument one can show that already $\mathrm{EA}^{2}+\Phi_{i t}+\Pi_{1}^{0}-\mathrm{AC}^{-}$proves the totality of the Ackermann function (see chapter 12 of [10] for details on this).

So any proof of conservation of systems based on $\Pi_{k}^{0}$-AC ${ }^{-}$over $\Sigma_{k}^{0}$-IA has to take into account carefully the structure of the functionals of type level 2 which are definable in the given system.

Things become of course even more complicated for the systems $G_{n} A^{\omega}+$ AC-qf $+\Delta$ instead of $\mathrm{EA}^{2}+\mathrm{AC}^{0,0}$-qf which we are treating in this paper. In particular we show the following result:

\footnotetext{
${ }^{4}$ Both aspects are not taken into account appropriately in [21] where $\Pi_{k}^{0}-\mathrm{CA}^{-}$and $\Pi_{k}^{0}-\mathrm{AC}^{-}$are studied systematically for the first time. As a consequence of this, theorems $5.8,5.13$ and corollaries 5.9,5.14 in [21] are not correct (see [11] and in particular chapter 12 of [10] for a thorough investigation of this matter).
} 
Let $t, \xi_{1}, \xi_{2}$ be closed terms of $\mathrm{G}_{n} \mathrm{~A}^{\omega}$ and $B: \equiv \forall u^{1} \forall v \leq_{\tau} t u B_{a r}(u, v)$ a sentence of $\mathrm{G}_{n} \mathrm{~A}^{\omega}$ where $B_{a r}(u, v) \in \Pi_{\infty}^{0}$. Then for every $k \in \mathbb{N}$ the following rule holds

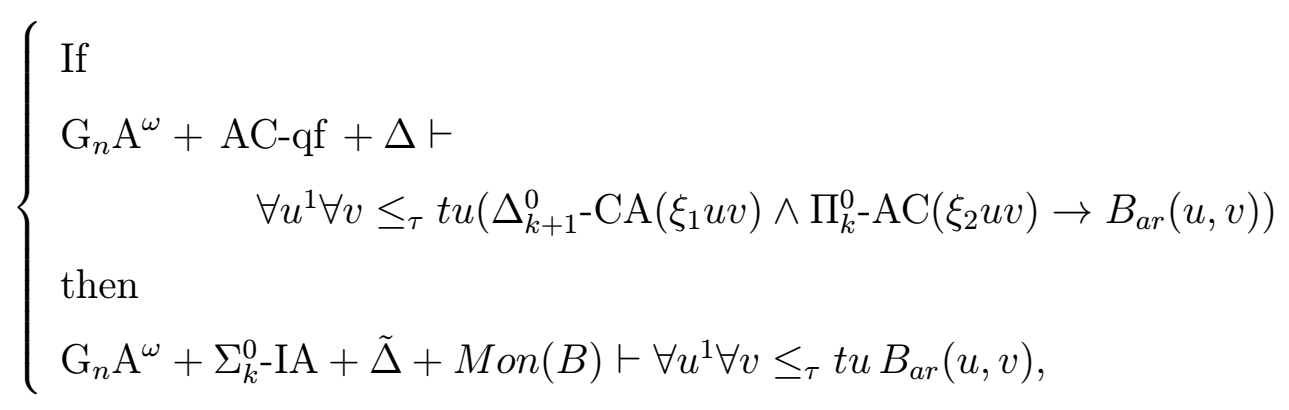

where $\operatorname{Mon}(B)$ expresses that $B$ is monotone in the sense of definition 2.3 below and

$\tilde{\Delta}:=\left\{\exists Y^{\rho \delta} \leq s \forall x^{\delta}, z^{\gamma} A_{0}(x, Y x, z): \forall x^{\delta} \exists y \leq_{\rho} s x \forall z^{\gamma} A_{0}(x, y, z) \in \Delta\right\}$.

( $\Delta$ and consequently $\tilde{\Delta}$ may be empty).

If $B_{a r} \in \Pi_{k+2}^{0}$, then the monotonicity assumption $\operatorname{Mon}(B)$ can be avoided, i.e. the conlusion can be strengthened to

$$
\mathrm{G}_{n} \mathrm{~A}^{\omega}+\Sigma_{k}^{0} \mathrm{IA}+\tilde{\Delta} \vdash \forall u^{1} \forall v \leq_{\tau} t u B_{a r}(u, v) .
$$

These results will be used also to prove new conservation results for $\mathrm{EA}+\Pi_{k}^{0}$-CP over EA $+\Sigma_{k}^{0}$-IA which strengthen the Friedman-Paris-Kirby result. ${ }^{5}$

Finally we consider generalizations $\Pi_{k}^{0} \mathrm{UB}^{-} \uparrow$ of the principle of uniform $\Sigma_{1}^{0}$ boundedness $\Sigma_{1}^{0}$-UB ${ }^{-}$which was studied in [12]. ${ }^{6}$ In [14] we showed that $\Sigma_{1}^{0}$ - $\mathrm{UB}^{-}$proves already relative to $\mathrm{G}_{2} \mathrm{~A}^{\omega}+\mathrm{AC}$-qf many important analytical theorems (like Dini's theorem, the attainment of the maximum for $f \in C\left([0,1]^{d}, \mathbb{R}\right)$, the sequential Heine-Borel property for $[0,1]^{d}$, the existence of an inverse function for every strictly monotone function $f \in C[0,1]$ and others) but does not contribute to the growth of extractable bounds, thereby guaranteing the extractability of polynomial bounds when applied in the context of $\mathrm{G}_{2} \mathrm{~A}^{\omega}+\mathrm{AC}-\mathrm{qf}$.

\footnotetext{
${ }^{5}$ A proof-theoretic treatment of the Friedman-Paris-Kirby result was first given in [21]. However the proof in [21] contains a serious gap. See [1] for a correction of Sieg's proof. Another proof-theoretic treatment can be found in [3].

${ }^{6}$ Whereas we generally use the superscript '-' to denote the restriction $S^{-}$of a schema $S$ to function parameter-free instances of $S$, this superscript has a different meaning in the context of principles of uniform boundedness. Although this might be troublesome we wish to stick to the notation for these principles from [12] where they were introduced.
} 
Whereas the straightforward generalization of $\Sigma_{1}^{0} \mathrm{UB}^{-}$to $\Pi_{k}^{0}$-formulas is inconsistent with $\mathrm{G}_{2} \mathrm{~A}^{\omega}$ already for $k=1$, our restricted version

$\Pi_{k}^{0}-\mathrm{UB}^{-} \uparrow$ ( introduced in the present paper) is consistent. In [15] we implicitly used (a special case of) $\Pi_{1}^{0}-\mathrm{UB}^{-} \wedge$ to prove the Bolzano-Weierstraß principle and the Ascoli-lemma and it were these proofs which were used to calibrate faithfully the arithmetical strength of these principles.

One of the results on $\Pi_{k}^{0}-\mathrm{UB}^{-} \uparrow$ to be proved in the present paper is that we may strengthen the assumption of the rule stated above by adding $\Pi_{k}^{0}-\mathrm{UB}^{-} \wedge\left(\xi_{3} u v\right)$ to $\Delta_{k+1}^{0} \mathrm{CA}\left(\xi_{1} u v\right) \wedge \Pi_{k}^{0}-\mathrm{AC}\left(\xi_{2} u v\right)$.

\section{Monotone formulas and their Skolem normal forms}

In this section we review some of the proof-theoretic tools from [13] on which the present paper is based and also recall some of the basic concepts and definitions from [12].

The set $\mathbf{T}$ of all finite types is defined inductively by

$$
\text { (i) } 0 \in \mathbf{T} \text { and (ii) } \rho, \tau \in \mathbf{T} \Rightarrow \tau(\rho) \in \mathbf{T} \text {. }
$$

Terms which denote a natural number have type 0. Elements of type $\tau(\rho)$ are functions which map objects of type $\rho$ to objects of type $\tau$.

The set $\mathbf{P} \subset \mathbf{T}$ of pure types is defined by

(i) $0 \in \mathbf{P}$ and $(i i) \rho \in \mathbf{P} \Rightarrow 0(\rho) \in \mathbf{P}$.

Brackets whose occurrences are uniquely determined are often omitted, e.g. we write $0(00)$ instead of $0(0(0))$. Furthermore we write for short $\tau \rho_{k} \ldots \rho_{1}$ instead of $\tau\left(\rho_{k}\right) \ldots\left(\rho_{1}\right)$. Pure types can be represented by natural numbers: $0(n):=n+1$. The types $0,00,0(00), 0(0(00)) \ldots$ are so represented by $0,1,2,3 \ldots$ For arbitrary types $\rho \in \mathbf{T}$ the degree of $\rho$ (for $\operatorname{short} \operatorname{deg}(\rho)$ ) is defined by $\operatorname{deg}(0):=0$ and $\operatorname{deg}(\tau(\rho)):=\max (\operatorname{deg}(\tau), \operatorname{deg}(\rho)+1)$. For pure types the degree is just the number which represents this type.

\section{Description of the theories $(E)-G_{n} A^{\omega}$}

Our theories $\mathcal{T}^{\omega}$ used in this paper are based on many-sorted classical logic formulated in the language of all finite types plus the combinators $\Pi_{\rho, \tau}, \Sigma_{\delta, \rho, \tau}$ which allow the definition of $\lambda$-abstraction. $\mathcal{T}_{i}^{\omega}$ denotes the intuitionistic variant of $\mathcal{T}^{\omega}$.

The systems $\mathrm{G}_{n} \mathrm{~A}^{\omega}$ (for all $n \geq 1$ ) are introduced in [12] to which we refer for details. $G_{n} A^{\omega}$ has as primitive relations $=_{0}, \leq_{0}$ for objects of type 0 , the 
constant $0^{0}$, functions $\min _{0}, \max _{0}, S^{00}$ (successor), $A_{0}, \ldots, A_{n}$, where $A_{i}$ is the $i$-th branch of the Ackermann function (i.e. $A_{0}(x, y)=y^{\prime}, A_{1}(x, y)=x+$ $\left.y, A_{2}(x, y)=x \cdot y, A_{3}(x, y)=x^{y}, \ldots\right)$, functionals of type level $2: \Phi_{1}, \ldots, \Phi_{n}$, where $\Phi_{1} f x=\max _{0}(f 0, \ldots, f x)$ and $\Phi_{i}$ is the iteration of $A_{i-1}$ on the $f$ values for $i \geq 2$, i.e. $\Phi_{2} f x=\sum_{i=0}^{x} f i, \Phi_{3} f x=\prod_{i=0}^{x} f i, \ldots$. We also have a bounded search functional $\mu_{b}$ and bounded predicative recursion provided by recursor constants $\tilde{R}_{\rho}$ (where 'predicative' means that recursion is possible only at the type-0-level as in the case of the (unbounded) Kleene-Feferman recursors $\widehat{R}_{\rho}$ ). Moreover $\mathrm{G}_{n} \mathrm{~A}^{\omega}$ contains a quantifier-free rule of extensionality $\mathrm{QF}-\mathrm{ER}$.

In addition to the defining axioms for the constants of our theories all true sentences having the form $\forall x^{\rho} A_{0}(x)$, where $A_{0}$ is quantifier-free and $\operatorname{deg}(\rho) \leq 2$, are added as axioms. By 'true' we refer to the full set-theoretic model $\mathcal{S}^{\omega}$. In given proofs however only very special universal axioms will be used which can be proved in suitable extensions of our theories. Nevertheless we include them all as axioms in order to emphasize that (proofs of) universal sentences do not contribute to the growth of extractable bounds. In particular this covers all instances of the schema of quantifier-free induction (The main results in this paper are also valid for the variant of $G_{n} A_{i}^{\omega}$ where the universal axioms are replaced by the schema of quantifier-free induction). The restriction $\operatorname{deg}(\rho) \leq 2$ has a technical reason discussed in [12].

$\mathrm{G}_{\infty} \mathrm{A}^{\omega}:=\bigcup_{n \in \mathbb{N}} \mathrm{G}_{n} \mathrm{~A}^{\omega}$.

$\mathrm{PA}^{\omega}, \mathrm{PA}_{i}^{\omega}$ are the extensions of $\mathrm{G}_{n} \mathrm{~A}^{\omega}, \mathrm{G}_{n} \mathrm{~A}_{i}^{\omega}$ obtained by the addition of the schema of full induction and all (impredicative) primitive recursive functionals in the sense of [5].

$\mathrm{E}-\mathcal{T}_{(i)}^{\omega}$ denotes the theory which results from $\mathcal{T}_{(i)}^{\omega}$ when the quantifier-free rule of extensionality is replaced by the axioms of extensionality (E)

$$
\forall x^{\rho}, y^{\rho}, z^{\tau \rho}\left(x={ }_{\rho} y \rightarrow z x={ }_{\tau} z y\right)
$$

for all finite types $\left(x={ }_{\rho} y\right.$ is defined as $\forall z_{1}^{\rho_{1}}, \ldots, z_{k}^{\rho_{k}}\left(x z_{1} \ldots z_{k}={ }_{0} y z_{1} \ldots z_{k}\right)$ where $\left.\rho=0 \rho_{k} \ldots \rho_{1}\right)$.

$\mathrm{G}_{n} \mathrm{R}^{\omega}$ and $T$ denote the sets of all closed terms of $(\mathrm{E})-\mathrm{G}_{n} \mathrm{~A}_{(i)}^{\omega}$ and $(\mathrm{E})-\mathrm{PA}_{(i)}^{\omega}$. $T_{k}$ is the subset of all closed terms of $T$ which contain the Gödel-recursors $R_{\rho}$ for $\rho$ of type level $\leq k$ only.

Definition 2.1 Between functionals of type $\rho$ we define relations $\leq_{\rho}$ ('less 
or equal') and $s-m a j_{\rho}$ ('strongly majorizes') by induction on the type:

$$
\begin{gathered}
\left\{\begin{array}{l}
x_{1} \leq_{0} x_{2}: \equiv\left(x_{1} \leq_{0} x_{2}\right), \\
x_{1} \leq_{\tau \rho} x_{2}: \equiv \forall y^{\rho}\left(x_{1} y \leq_{\tau} x_{2} y\right) ;
\end{array}\right. \\
\left\{\begin{array}{l}
x^{*} s-m a j_{0} x: \equiv x^{*} \geq_{0} x, \\
x^{*} s-m a j_{\tau \rho} x: \equiv \forall y^{* \rho}, y^{\rho}\left(y^{*} s-m a j_{\rho} y \rightarrow x^{*} y^{*} s-m a j_{\tau} x^{*} y, x y\right) .
\end{array}\right.
\end{gathered}
$$

Remark 2.2 's-maj' is a variant of W.A. Howard's relation 'maj' from [6] which is due to [2]. For more details see [8].

Let $A(\underline{a})$ be a formula of $\mathrm{G}_{n} \mathrm{~A}^{\omega}$ ( $\underline{a}$ are all free variables of $A$ ) and $\exists \underline{x} \forall \underline{y} A_{D}(\underline{x}, \underline{y}, \underline{a})$ its Gödel functional interpretation (see e.g. [24] for details on Gödel's functional interpretation). We say that a tuple of closed terms $\underline{t}$ realizes the monotone functional interpretation of $A(\underline{a})$ if $^{7}$

$$
(*) \exists \underline{x}\left(\underline{t} \mathrm{~s}-\mathrm{maj} \underline{x} \wedge \forall \underline{a}, \underline{y} A_{D}(\underline{x} \underline{a}, \underline{y}, \underline{a})\right)
$$

(Monotone functional interpretation which directly extracts a tuple $\underline{t}$ satisfying $(*)$ from a proof of $A(\underline{a})$ was introduced in [9]. See also [12] for details.)

Definition 2.3 ([13]) Let $A \in \mathcal{L}\left(G_{n} A^{\omega}\right)$ be a formula having the form

$$
A \equiv \forall u^{1} \forall v \leq_{\tau} t u \exists y_{1}^{0} \forall x_{1}^{0} \ldots \exists y_{k}^{0} \forall x_{k}^{0} \exists w^{\gamma} A_{0}\left(u, v, y_{1}, x_{1}, \ldots, y_{k}, x_{k}, w\right),
$$

where $A_{0}$ is quantifier-free and contains only $u, v, \underline{y}, \underline{x}, w$ free, $t \in G_{n} R^{\omega}$ and $\tau, \gamma$ are arbitrary finite types.

1) A is called (arithmetically) monotone if

$$
\begin{aligned}
& \operatorname{Mon}(A): \equiv \\
& \left\{\begin{array}{c}
\forall u^{1} \forall v \leq_{\tau} t u \forall x_{1}, \tilde{x}_{1}, \ldots, x_{k}, \tilde{x}_{k}, y_{1}, \tilde{y}_{1}, \ldots y_{k}, \tilde{y}_{k} \\
\left(\bigwedge_{i=1}^{k}\left(\tilde{x}_{i} \leq_{0} x_{i} \wedge \tilde{y}_{i} \geq_{0} y_{i}\right) \wedge \exists w^{\gamma} A_{0}\left(u, v, y_{1}, x_{1}, \ldots, y_{k}, x_{k}, w\right)\right. \\
\left.\rightarrow \exists w^{\gamma} A_{0}\left(u, v, \tilde{y}_{1}, \tilde{x}_{1}, \ldots, \tilde{y}_{k}, \tilde{x}_{k}, w\right)\right) .
\end{array}\right.
\end{aligned}
$$

\footnotetext{
${ }^{7}$ Here $\underline{t} \mathrm{~s}-\mathrm{maj} \underline{x}$ means $\bigwedge_{i}\left(t_{i} \mathrm{~s}-\mathrm{maj} x_{i}\right)$.
} 
2) The Herbrand normal form $A^{H}$ of $A$ is defined to be

$$
\begin{aligned}
& A^{H}: \equiv \forall u^{1} \forall v \leq_{\tau} t u \forall h_{1}^{\rho_{1}}, \ldots, h_{k}^{\rho_{k}} \exists y_{1}^{0}, \ldots, y_{k}^{0}, w^{\gamma} \\
& \underbrace{A_{0}\left(u, v, y_{1}, h_{1} y_{1}, \ldots, y_{k}, h_{k} y_{1} \ldots y_{k}, w\right)}_{A_{0}^{H}: \equiv}, \\
& \text { where } \rho_{i}=0 \underbrace{(0) \ldots(0)}_{i} .
\end{aligned}
$$

Remark 2.4 In definition 2.3 (and theorems 2.5,2.7 below) one may also have tuples ' $\exists \underline{w}$ ' instead of ' $\exists w^{\gamma}$ ' in $A$ where $\underline{w}=w_{1}^{\gamma_{1}}, \ldots, w_{l}^{\gamma_{l}}$ and $\gamma_{i}$ is arbitrary. Also instead of $\forall u^{1}$ we may have $\forall \underline{u}$ where $\underline{u}=u_{1}^{\rho_{1}}, \ldots, u_{q}^{\rho_{q}}$ with $\operatorname{deg}\left(\rho_{i}\right) \leq 1$ for $1 \leq i \leq q$. In particular we can consider an innermost existential number quantifier $\exists y_{k+1}^{0}$ as part of $\exists \underline{w}$ and an outermost universal number quantifier $\forall x_{0}^{0}$ as part of $\forall \underline{u}$. So for $\forall x_{0}^{0}$ and $\exists y_{k+1}^{0}$ no monotonicity is required in definition 2.3.1).

Theorem 2.5 ([13]) Let $n \geq 1$ and $\Psi_{1}, \ldots, \Psi_{k} \in G_{n} R^{\omega}$. Then

$$
\begin{aligned}
G_{n} A^{\omega}+\operatorname{Mon}(A) \vdash \forall u^{1} \forall v & \leq_{\tau} t u \forall h_{1}, \ldots, h_{k}\left(\bigwedge_{i=1}^{k}\left(h_{i} \text { monotone }\right)\right. \\
& \left.\rightarrow \exists y_{1} \leq_{0} \Psi_{1} u \underline{h} \ldots \exists y_{k} \leq_{0} \Psi_{k} u \underline{h} \exists w^{\gamma} A_{0}^{H}\right) \rightarrow A,
\end{aligned}
$$

where $\left(h_{i}\right.$ monotone $): \equiv \forall x_{1}, \ldots, x_{i}, y_{1}, \ldots, y_{i}\left(\bigwedge_{j=1}^{i}\left(x_{j} \geq_{0} y_{j}\right) \rightarrow h_{i} \underline{x} \geq_{0} h_{i} \underline{y}\right)$.

Definition 2.6 (Bounded choice) $b-A C:=\bigcup_{\delta, \rho \in \mathbf{T}}\left\{\left(b-A C^{\delta, \rho}\right)\right\}$ denotes the schema of bounded choice

$$
\left(b-A C^{\delta, \rho}\right): \forall Z^{\rho \delta}\left(\forall x^{\delta} \exists y \leq_{\rho} Z x A(x, y, Z) \rightarrow \exists Y \leq_{\rho \delta} Z \forall x A(x, Y x, Z)\right) .
$$

In general $\mathrm{G}_{n} \mathrm{~A}^{\omega} \vdash A^{H}$ does not imply $\mathrm{G}_{n} \mathrm{~A}^{\omega} \vdash A$ (see [13] for a detailed discussion of this phenomenon), which is in contrast to the first-order case where the derivability of $A^{H}$ follows from that of $A$ by Herbrand's theorem (see [20]). If however $A$ is monotone then this rule is valid also for $\mathrm{G}_{n} \mathrm{~A}^{\omega}$ (but for very different reasons):

Theorem 2.7 ([13]) Let $A$ be as in thm.2.5 and $\Delta$ be a set of sentences $\forall x^{\delta} \exists y \leq_{\rho} s x \forall z^{\eta} G_{0}(x, y, z)$ where $s$ is a closed term of $G_{n} A^{\omega}$ and $G_{0}$ a 
quantifier-free formula, and let $A^{\prime}$ denote the negative translation ${ }^{8}$ of $A$. Then the following rule holds:

$$
\left\{\begin{array}{l}
G_{n} A^{\omega}+A C-q f+\Delta \vdash A^{H} \wedge M o n(A) \Rightarrow \\
G_{n} A^{\omega}+\tilde{\Delta} \vdash A \text {, and by monotone functional interpretation one can } \\
\text { extract a tuple } \underline{\Psi} \in G_{n} R^{\omega} \text { such that } \\
G_{n} A_{i}^{\omega}+\tilde{\Delta} \vdash \underline{\Psi} \text { satisfies the monotone functional interpretation of } A^{\prime},
\end{array}\right.
$$

where $\tilde{\Delta}:=\left\{\exists Y \leq_{\rho \delta} s \forall x^{\delta}, z^{\eta} G_{0}(x, Y x, z): \forall x^{\delta} \exists y \leq_{\rho} s x \forall z^{\eta} G_{0}(x, y, z) \in \Delta\right\}$. (In particular the second conclusion can be proved in $G_{n} A_{i}^{\omega}+\Delta+b-A C$ ).

The weakened conclusion $G_{n} A^{\omega}+\tilde{\Delta}+\operatorname{Mon}(A) \vdash A$ follows already from $G_{n} A^{\omega}+A C-q f+\Delta \vdash A^{H}$. $^{9}$

\section{Making arithmetical comprehension monotone}

In this section we consider the arithmetical content of instances $\Pi_{k}^{0}-\mathrm{CA}(\xi u v)$ of $\Pi_{k}^{0}$-CA which are used in given proofs of sentences $\forall u^{1} \forall v \leq_{\tau} t u B_{a r}(u, v)$ as discussed in the introduction.

\section{Definition 3.1}

$$
\Pi_{k}^{0}-C A(f): \equiv \exists g^{1} \forall x^{0}\left(g x={ }_{0} 0 \leftrightarrow \forall u_{1}^{0} \exists u_{2}^{0} \ldots \exists^{(d)} u_{k}^{0}\left(f(x, \underline{u})={ }_{0} 0\right)\right) .{ }^{10}
$$

Remark 3.2 There is no need here to incorporate closure under number parameters in the definition of $\Pi_{k}^{0}-C A(f)$, i.e. by defining

$$
\Pi_{k}^{0}-C A(f): \equiv \forall l^{0} \exists g^{1} \forall x^{0}\left(g x={ }_{0} 0 \leftrightarrow \forall u_{1}^{0} \exists u_{2}^{0} \ldots \exists^{(d)} u_{k}^{0}\left(f(l, x, \underline{u})={ }_{0} 0\right)\right),
$$

since the latter can be reduced to the former (relative to $G_{n} A^{\omega}$ for $n \geq 2$ ) by coding $l, x$ together and applying comprehension without number parameters to this pair.

\footnotetext{
${ }^{8}$ Here we can use Gödel's [4] translation or any other of the various negative translations. For a systematical treatment of negative translations see [16].

${ }^{9}$ This last assertion is not stated in the formulation of the theorem in [13] but does follow immediately from its proof.

${ }^{10}$ Whether one has here ' $\exists u_{k}^{0}$ ' or ' $\forall u_{k}^{0}$ ' depends of course on whether $k$ is even or odd.
} 
In order to be able to apply the method of elimination of Skolem functions for monotone formulas from section 2 we follow this strategy:

Construct an arithmetical principle $A_{a r}(f)$ such that for suitable $\xi_{1}, \xi_{2} \in$ $\mathrm{G}_{n} \mathrm{R}^{\omega}$ :

1) $\mathrm{G}_{n} \mathrm{~A}^{\omega} \vdash \operatorname{Mon}\left(\forall f A_{a r}(f)\right)$,

2) $\mathrm{G}_{n} \mathrm{~A}^{\omega} \vdash \forall f\left(A_{a r}^{S}\left(\xi_{1} f\right) \rightarrow \Pi_{k}^{0}-\mathrm{CA}(f)\right)$ and

3) $\mathrm{G}_{n} \mathrm{~A}^{\omega} \vdash \forall f\left(\Pi_{k}^{0}-\mathrm{CA}\left(\xi_{2} f\right) \rightarrow A_{a r}(f)\right)$.

Because of 2) the use of $\Pi_{k}^{0}-\mathrm{CA}(\xi u v)$ in a given proof of a monotone sentence $\forall u^{1} \forall v \leq_{\tau} t u B_{a r}(u, v)$ can be reduced to the use of $A_{a r}^{S}\left(\xi^{\prime} u v\right)$ (where $\xi^{\prime} u v:=$ $\left.\xi_{1}(\xi u v)\right)$ which in turn (by 1) and theorem 2.7) can be reduced to the use of $A_{a r}\left(\xi^{\prime} u v\right)$. Because of 3$)$ nothing is lost by this reduction.

It will turn out that the correct principle $A_{a r}(f)$ is a 'monotone version' $\Pi_{k}^{0}-\mathrm{TND}^{\text {mon }}(f)$ of the tertium-non-datur principle for $\Pi_{k}^{0}$-formulas.

Definition 3.3 In the following $m:=\frac{k}{2}$ if $k$ is even (resp. $m:=\frac{k-1}{2}$ if $k$ is odd).

1) The $\Pi_{k}^{0}$-tertium-non-datur axiom is given by the following formula (where $f$ is a function variable of appropriate type) $)^{11}$

$$
\begin{aligned}
& \underline{\Pi_{k}^{0}-T N D}(f): \equiv \\
& \left\{\begin{array}{l}
\forall x^{0}\left(\forall y_{1}^{0} \exists z_{1}^{0} \ldots \forall y_{m}^{0} \exists z_{m}^{0}\left(\forall y_{m+1}^{0}\right)\left(f\left(x, y_{1}, z_{1}, \ldots, y_{m}, z_{m},\left(y_{m+1}\right)\right)={ }_{0} 0\right)\right. \\
\left.\vee \exists u_{1}^{0} \forall v_{1}^{0} \ldots \exists u_{m}^{0} \forall v_{m}^{0}\left(\exists u_{m+1}^{0}\right)\left(f\left(x, u_{1}, v_{1}, \ldots, u_{m}, v_{m},\left(u_{m+1}\right)\right) \neq 0\right)\right),
\end{array}\right.
\end{aligned}
$$

2) We also need the following prenex normal form of $\Pi_{k}^{0}-T N D(f)$ :

$$
\begin{aligned}
& \frac{\Pi_{k}^{0}-T N D}{(f)^{p r}: \equiv} \\
& \left\{\begin{array}{c}
\forall x^{0} \exists u_{1}^{0} \forall y_{1}^{0} \exists z_{1}^{0} \forall v_{1}^{0} \ldots \exists u_{m}^{0} \forall y_{m}^{0} \exists z_{m}^{0} \forall v_{m}^{0}\left(\exists u_{m+1}^{0} \forall y_{m+1}^{0}\right) \\
\left(f\left(x, y_{1}, z_{1}, \ldots, y_{m}, z_{m},\left(y_{m+1}\right)\right)={ }_{0} 0 \vee\right. \\
\left.f\left(x, u_{1}, v_{1}, \ldots, u_{m}, v_{m},\left(u_{m+1}\right)\right) \neq 0\right),
\end{array}\right.
\end{aligned}
$$

\footnotetext{
${ }^{11}$ Here and in the following the quantifiers $\forall y_{m+1}^{0}, \exists u_{m+1}^{0}$ are only present if $k$ is odd.
} 
3) The Skolem normal form of $\Pi_{k}^{0}-T N D(f)^{p r}$ is given by

$$
\begin{aligned}
& \underline{\left(\Pi_{k}^{0}-T N D(f)^{p r}\right)^{S}}: \equiv \\
& \left\{\begin{array}{l}
\exists h_{1}, \ldots, h_{m},\left(h_{m+1}\right), g_{1}, \ldots, g_{m} \forall x^{0}, y_{1}^{0}, v_{1}^{0}, \ldots, y_{m}^{0}, v_{m}^{0},\left(y_{m+1}\right) \\
\left(f\left(x, y_{1}, g_{1}\left(x, y_{1}\right), \ldots, y_{m}, g_{m}\left(x, y_{1}, \ldots, y_{m}, v_{1}, \ldots, v_{m-1}\right),\left(y_{m+1}\right)\right)={ }_{0} 0 \vee\right. \\
f\left(x, h_{1} x, v_{1}, \ldots, h_{m}\left(x, y_{1}, \ldots, y_{m-1}, v_{1}, \ldots, v_{m-1}\right), v_{m},\right. \\
\left.\left.\quad\left(h_{m+1}\left(x, y_{1}, \ldots, y_{m}, v_{1}, \ldots, v_{m}\right)\right)\right) \neq 0\right) .
\end{array}\right.
\end{aligned}
$$

Remark 3.4 For $n \geq 2$ we have coding of finite tuples (of fixed length) available in $G_{n} A^{\omega}$. Hence quantifier-blocks can be contracted to a single quantifier. Since in all of our results we assume that (at least) $n \geq 2$, it is no restriction in the definition above to consider only single quantifiers.

Lemma 3.5 For every $k \in \mathbb{N}$ the following implication can be proved in $G_{1} A^{\omega}$ :

$$
\forall f\left(\left(\Pi_{k}^{0}-T N D(f)^{p r}\right)^{S} \rightarrow \Pi_{k}^{0}-C A(f)\right)
$$

\section{Proof:}

For notational simplicity we confine ourselves to the case $k=4$ which well shows the general pattern of the proof for arbitrary $k$ :

$\left(\Pi_{4}^{0} \text {-TND }(f)^{p r}\right)^{S}$ yields the existence of functions $g_{1}, g_{2}, h_{1}, h_{2}$ such that

$$
\text { (1) }\left\{\begin{array}{r}
\forall x, y_{1}, v_{1}, y_{2}\left(f\left(x, y_{1}, g_{1}\left(x, y_{1}\right), y_{2}, g_{2}\left(x, y_{1}, y_{2}, v_{1}\right)\right)=0\right. \\
\left.\vee \forall v_{2}\left(f\left(x, h_{1} x, v_{1}, h_{2}\left(x, y_{1}, v_{1}\right), v_{2}\right) \neq 0\right)\right) .
\end{array}\right.
$$

(1) in turn yields

$$
\begin{gathered}
\text { (2) }\left\{\begin{array}{r}
\forall x, y_{1}, v_{1}\left(\forall y_{2} \exists z_{2} f\left(x, y_{1}, g_{1}\left(x, y_{1}\right), y_{2}, z_{2}\right)=0\right. \\
\left.\vee \forall v_{2}\left(f\left(x, h_{1} x, v_{1}, h_{2}\left(x, y_{1}, v_{1}\right), v_{2}\right) \neq 0\right)\right),
\end{array}\right. \\
\text { (3) }\left\{\begin{array}{r}
\forall x, y_{1}, v_{1}\left(\forall y_{2} \exists z_{2} f\left(x, y_{1}, g_{1}\left(x, y_{1}\right), y_{2}, z_{2}\right)=0\right. \\
\left.\vee \exists u_{2} \forall v_{2}\left(f\left(x, h_{1} x, v_{1}, u_{2}, v_{2}\right) \neq 0\right)\right),
\end{array}\right. \\
\left\{\begin{array}{r}
\forall x, y_{1}\left(\forall y_{2} \exists z_{2} f\left(x, y_{1}, g_{1}\left(x, y_{1}\right), y_{2}, z_{2}\right)=0\right. \\
\left.\vee \forall v_{1} \exists u_{2} \forall v_{2}\left(f\left(x, h_{1} x, v_{1}, u_{2}, v_{2}\right) \neq 0\right)\right),
\end{array}\right.
\end{gathered}
$$


(5) $\left\{\begin{array}{c}\forall x, y_{1}\left(\exists z_{1} \forall y_{2} \exists z_{2} f\left(x, y_{1}, z_{1}, y_{2}, z_{2}\right)=0\right. \\ \left.\vee \forall v_{1} \exists u_{2} \forall v_{2}\left(f\left(x, h_{1} x, v_{1}, u_{2}, v_{2}\right) \neq 0\right)\right)\end{array}\right.$

and finally

(6) $\forall x\left(\forall y_{1} \exists z_{1} \forall y_{2} \exists z_{2} f\left(x, y_{1}, z_{1}, y_{2}, z_{2}\right)=0 \vee \forall v_{1} \exists u_{2} \forall v_{2}\left(f\left(x, h_{1} x, v_{1}, u_{2}, v_{2}\right) \neq 0\right)\right)$.

(1) applied to $y_{1}:=h_{1} x, v_{1}:=g_{1}\left(x, h_{1} x\right), y_{2}:=h_{2}\left(x, h_{1} x, g_{1}\left(x, h_{1} x\right)\right)$ gives

$(*): \equiv$

$$
\begin{gathered}
\forall x^{0}\left(f \left(x, h_{1} x, g_{1}\left(x, h_{1} x\right), h_{2}\left(x, h_{1} x, g_{1}\left(x, h_{1} x\right)\right), g_{2}\left(x, h_{1} x, h_{2}\left(x, h_{1} x, g_{1}\left(x, h_{1} x\right)\right),\right.\right.\right. \\
\left.\left.\left.g_{1}\left(x, h_{1} x\right)\right)\right)=0 \vee \forall v_{2}\left(f\left(x, h_{1} x, g_{1}\left(x, h_{1} x\right), h_{2}\left(x, h_{1} x, g_{1}\left(x, h_{1} x\right)\right), v_{2}\right) \neq 0\right)\right) .
\end{gathered}
$$

We now show $(+): \equiv$

$\forall x^{0}\left(f\left(x, h_{1} x, g_{1}\left(x, h_{1} x\right), h_{2}\left(x, h_{1} x, g_{1}\left(x, h_{1} x\right)\right), g_{2}\left(x, h_{1} x, h_{2}\left(x, h_{1} x, g_{1}\left(x, h_{1} x\right)\right)\right.\right.\right.$,

$\left.\left.\left.g_{1}\left(x, h_{1} x\right)\right)\right)=0 \leftrightarrow \forall y_{1} \exists z_{1} \forall y_{2} \exists z_{2}\left(f\left(x, y_{1}, z_{1}, y_{2}, z_{2}\right)=0\right)\right)$.

$(+)$ yields the claim of the lemma with

$$
\begin{aligned}
& g x:=\Phi x h_{1} h_{2} g_{1} g_{2}:= \\
& \left\{\begin{array}{r}
f\left(x, h_{1} x, g_{1}\left(x, h_{1} x\right), h_{2}\left(x, h_{1} x, g_{1}\left(x, h_{1} x\right)\right),\right. \\
\left.\quad g_{2}\left(x, h_{1} x, h_{2}\left(x, h_{1} x, g_{1}\left(x, h_{1} x\right)\right), g_{1}\left(x, h_{1} x\right)\right)\right) .
\end{array}\right.
\end{aligned}
$$

Proof of $(+)$ :

' $\rightarrow$ ': $\Phi x f h_{1} h_{2} g_{1} g_{2}=0$ implies

$$
\neg \forall v_{2}\left(f\left(x, h_{1} x, g_{1}\left(x, h_{1} x\right), h_{2}\left(x, h_{1} x, g_{1}\left(x, h_{1} x\right)\right), v_{2}\right) \neq 0\right) .
$$

Hence by (2) (putting $y_{1}:=h_{1} x, v_{1}:=g_{1}\left(x, h_{1} x\right)$ )

$$
\forall y_{2} \exists z_{2}\left(f\left(x, h_{1} x, g_{1}\left(x, h_{1} x\right), y_{2}, z_{2}\right)=0\right)
$$

and therefore

$$
\exists z_{1} \forall y_{2} \exists z_{2}\left(f\left(x, h_{1} x, z_{1}, y_{2}, z_{2}\right)=0\right),
$$

i.e.

$$
\neg \forall v_{1} \exists u_{2} \forall v_{2}\left(f\left(x, h_{1} x, v_{1}, u_{2}, v_{2}\right) \neq 0\right) .
$$

By (6) this implies

$$
\forall y_{1} \exists z_{1} \forall y_{2} \exists z_{2}\left(f\left(x, y_{1}, z_{1}, y_{2}, z_{2}\right)=0\right) .
$$

' $\leftarrow$ ': $\Phi x f h_{1} h_{2} g_{1} g_{2} \neq 0$ implies by $(*)$

$$
\forall v_{2}\left(f\left(x, h_{1} x, g_{1}\left(x, h_{1} x\right), h_{2}\left(x, h_{1} x, g_{1}\left(x, h_{1} x\right)\right), v_{2}\right) \neq 0\right)
$$


and therefore

$$
\exists u_{2} \forall v_{2}\left(f\left(x, h_{1} x, g_{1}\left(x, h_{1} x\right), u_{2}, v_{2}\right) \neq 0\right),
$$

i.e.

$$
\neg \forall y_{2} \exists z_{2}\left(f\left(x, h_{1} x, g_{1}\left(x, h_{1} x\right), y_{2}, z_{2}\right)=0\right) .
$$

By (4) this yields (putting $y_{1}:=h_{1} x$ )

$$
\forall v_{1} \exists u_{2} \forall v_{2}\left(f\left(x, h_{1} x, v_{1}, u_{2}, v_{2}\right) \neq 0\right)
$$

and therefore

$$
\exists u_{1} \forall v_{1} \exists u_{2} \forall v_{2}\left(f\left(x, u_{1}, v_{1}, u_{2}, v_{2}\right) \neq 0\right),
$$

which concludes the proof of $(+)$ and hence of the lemma.

Definition 3.6 For $a \Pi_{k}^{0}$-formula

$A(\underline{a}) \equiv \forall x_{1}^{0} \exists x_{2}^{0} \ldots \exists^{(d)} x_{k}^{0} A_{0}\left(\underline{a}, x_{1}, x_{2}, \ldots, x_{k}\right)$ of $G_{n} A^{\omega}$ (where $\underline{a}$ are all free variables of $A$ which may have arbitrary type) we define

$\tilde{A}(\underline{a}): \equiv$

$\forall x_{1}^{0} \exists x_{2}^{0} \ldots \exists^{(d)} x_{k}^{0} \forall \tilde{x}_{1} \leq x_{1} \exists \tilde{x}_{2} \leq x_{2} \ldots \exists^{(d)} \tilde{x}_{k} \leq x_{k} A_{0}\left(\underline{a}, \tilde{x}_{1}, \tilde{x}_{2}, \ldots, \tilde{x}_{k}\right)$.

In the following we need a variant Mon* of Mon where monotonicity is required for all number quantifiers (compare this with remark 2.4):

Definition 3.7 Let $A(\underline{a}): \equiv \forall x_{1}^{0} \exists y_{1}^{0} \ldots \forall x_{k}^{0} \exists y_{k}^{0} A_{0}\left(\underline{a}, x_{1}, y_{1}, \ldots, x_{k}, y_{k}\right){ }^{12}$ Then

$$
\begin{array}{r}
\operatorname{Mon}^{*}(A(\underline{a})): \equiv \forall x_{1}, \tilde{x}_{1}, y_{1}, \tilde{y}_{1}, \ldots, x_{k}, \tilde{x}_{k}, y_{k}, \tilde{y}_{k}\left(\bigwedge_{i=1}^{k}\left(\tilde{x}_{i} \leq_{0} x_{i} \wedge \tilde{y}_{i} \geq_{0} y_{i}\right) \rightarrow\right. \\
\left(A_{0}\left(\underline{a}, x_{1}, y_{1}, \ldots, x_{k}, y_{k}\right) \rightarrow A_{0}\left(\underline{a}, \tilde{x}_{1}, \tilde{y}_{1}, \ldots, \tilde{x}_{k}, \tilde{y}_{k}\right)\right) .
\end{array}
$$

Lemma 3.8 For $\tilde{A}(\underline{a})$ as in the previous definition we have

$$
G_{n} A^{\omega} \vdash \operatorname{Mon}^{*}(\tilde{A}(\underline{a})) \text {. }
$$

Proof: Trivial.

The $\Pi_{k}^{0}$-collection principle is the schema

$$
\underline{\Pi_{k}^{0} \text {-CP }}: \forall x \leq_{0} a \exists y^{0} A(x, y) \rightarrow \exists z^{0} \forall x \leq_{0} a \exists y \leq_{0} z A(x, y),
$$

for all $\Pi_{k}^{0}$-formulas $A(x, y)$.

\footnotetext{
${ }^{12}$ Here the quantifiers $\forall x_{1}^{0}$ and $\exists y_{k}^{0}$ may be empty ('dummy') quantifiers.
} 
Convention 3.9 In $\Pi_{k}^{0}-C P$ (and other axiom schemas which we will consider below) $A(x, y)$ may contain arbitrary parameters (besides $x, y$ ) of the language we consider. E.g. if we write $G_{n} A^{\omega}+\Pi_{k}^{0}-C P$ then instances of $\Pi_{k}^{0}-C P$ may contain parameters of arbitrary type. In $E A+\Pi_{k}^{0}-C P$ however (where EA denotes first-order elementary recursive arithmetic) instances of $\Pi_{k}^{0}-C P$ of course contain only number parameters.

$\Pi_{k}^{0}$-CP is equivalent over many systems (e.g. $\mathrm{G}_{n} \mathrm{~A}^{\omega}$ for $n \geq 3$ ) to the axiom schema of finite choice for $\Pi_{k}^{0}$-formulas

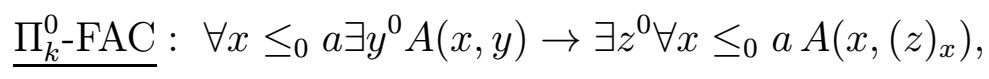

for all $\Pi_{k}^{0}$-formulas $A(x, y)$ (with the convention stated above).

In the presence of function variables as in $\mathrm{G}_{n} \mathrm{~A}^{\omega}$ the schema $\Pi_{k}^{0}$-CP can be expressed as a single second-order axiom $\forall f \Pi_{k}^{0}$-CP $(f)$, where

$\underline{\Pi_{k}^{0}-\mathrm{CP}(f)}: \equiv\left\{\begin{array}{l}\forall l^{0}, a^{0}\left(\forall x \leq_{0} a \exists y^{0} \forall u_{1}^{0} \exists u_{2}^{0} \ldots \exists^{(d)} u_{k}^{0}\left(f(l, a, x, y, \underline{u})={ }_{0} 0\right)\right. \\ \left.\rightarrow \exists z^{0} \forall x \leq_{0} a \exists y \leq_{0} z \forall u_{1}^{0} \exists u_{2}^{0} \ldots \exists^{(d)} u_{k}^{0}\left(f(l, a, x, y, \underline{u})={ }_{0} 0\right)\right) .\end{array}\right.$

By incorporating the universal closure w.r.t. to arithmetical parameters $\forall l^{0}, a^{0}$ in $\Pi_{k}^{0}-\mathrm{CP}(f)$, we achieve that the universal closure of every instance of $\Pi_{k}^{0}$-CP which contains only number parameters can be written as a sentence $\Pi_{k}^{0}-\mathrm{CP}(\xi)$ in $\mathrm{G}_{n} \mathrm{~A}^{\omega}$ where $\xi$ is a closed term (essentially the characteristic function of the quantifier-free matrix of the $\Pi_{k}^{0}$-formula $\left.A(x, y)\right)$ which will be of importance below.

The same is true for the principle of $\Sigma_{k}^{0}$-induction $\Sigma_{k}^{0}$-IA $(f)$ which we need below:

$$
\underline{\Sigma_{k}^{0}-\mathrm{IA}(f)}: \equiv\left\{\begin{array}{c}
\forall l^{0}\left(\exists u_{1}^{0} \forall u_{2}^{0} \ldots \forall^{(d)} u_{k}^{0}\left(f(l, 0, \underline{u})={ }_{0} 0\right) \wedge\right. \\
\forall x^{0}\left(\exists u_{1}^{0} \forall u_{2}^{0} \ldots \forall^{(d)} u_{k}^{0}\left(f(l, x, \underline{u})={ }_{0} 0\right) \rightarrow\right. \\
\left.\exists u_{1}^{0} \forall u_{2}^{0} \ldots \forall^{(d)} u_{k}^{0}\left(f\left(l, x^{\prime}, \underline{u}\right)={ }_{0} 0\right)\right) \\
\left.\rightarrow \forall x^{0} \exists u_{1}^{0} \forall u_{2}^{0} \ldots \forall^{(d)} u_{k}^{0}\left(f(l, x, \underline{u})={ }_{0} 0\right)\right) .
\end{array}\right.
$$

Lemma 3.10 Let $A(\underline{a}), \tilde{A}(\underline{a})$ be as in definition 3.6. Then for suitable $\xi_{1}, \ldots \xi_{l}, \tilde{\xi}_{1}, \ldots, \tilde{\xi}_{\tilde{l}} \in G_{n} R^{\omega}$ the following holds:

$$
G_{n} A^{\omega} \vdash \bigwedge_{i=1}^{l} \Pi_{k-2}^{0} C P\left(\xi_{i} \underline{a}\right) \rightarrow(A(\underline{a}) \rightarrow \tilde{A}(\underline{a}))
$$


and

$$
G_{n} A^{\omega} \vdash \bigwedge_{i=1}^{\tilde{l}} \Pi_{k-3}^{0}-C P\left(\tilde{\xi}_{i} \underline{a}\right) \rightarrow(\tilde{A}(\underline{a}) \rightarrow A(\underline{a}))
$$

(Here and in the following we use the convention that $\Pi_{k}^{0}-S$ is empty (i.e. $\equiv(0=0)$ for an axiom schema $S$ if $k<0)$.

Proof: Induction on $k$ : For $k=0,1$ the lemma is trivial. So let $k \geq 1$. $k \mapsto k+1:$ Consider

$$
A(\underline{a}) \equiv \forall x_{1}^{0} \exists x_{2}^{0} \ldots \exists^{(d)} x_{k+1}^{0} A_{0}\left(\underline{a}, x_{1}, x_{2}, \ldots, x_{k+1}\right) \in \Pi_{k+1}^{0} .
$$

By the induction hypothesis applied to the $\Pi_{k}^{0}$-formula

$$
\forall x_{2} \exists x_{3} \ldots \forall^{(d)} x_{k+1} \neg A_{0}\left(\underline{a}, x_{1}, \ldots, x_{k+1}\right)
$$

we have instances $\Pi_{k-2}^{0}-\mathrm{CP}\left(\xi_{i} \underline{a}\right)$ (note that instances of $\Pi_{k-3}^{0}-\mathrm{CP}$ can be considered as instance of $\Pi_{k-2}^{0}-\mathrm{CP}$ as well) such that $\bigwedge_{i} \Pi_{k-2}^{0}-\mathrm{CP}\left(\xi_{i} \underline{a}\right)$ implies (relative to $\mathrm{G}_{n} \mathrm{~A}^{\omega}$

$$
\begin{aligned}
& \exists x_{2} \forall x_{3} \ldots \exists^{(d)} x_{k+1} A_{0} \leftrightarrow \\
& \exists x_{2} \forall x_{3} \ldots \exists^{(d)} x_{k+1} \exists \tilde{x}_{2} \leq x_{2} \forall \tilde{x}_{3} \leq x_{3} \ldots A_{0}\left(\underline{a}, x_{1}, \tilde{x}_{2}, \ldots, \tilde{x}_{k+1}\right) .
\end{aligned}
$$

Hence

$$
\begin{aligned}
& A(\underline{a}) \\
& \leftrightarrow \forall x_{1} \exists x_{2} \ldots \exists^{(d)} x_{k+1} \exists \tilde{x}_{2} \leq x_{2} \ldots \exists^{(d)} \tilde{x}_{k+1} \leq x_{k+1} A_{0}\left(\underline{a}, x_{1}, \tilde{x}_{2}, \ldots, \tilde{x}_{k+1}\right) \\
& \leftrightarrow \forall x_{1} \forall \tilde{x}_{1} \leq x_{1} \exists x_{2} \ldots \exists^{(d)} x_{k+1} \exists \tilde{x}_{2} \leq x_{2} \ldots A_{0}\left(\underline{a}, \tilde{x}_{1}, \tilde{x}_{2}, \ldots, \tilde{x}_{k+1}\right) \\
& \stackrel{\Pi_{k-1}^{0}{ }^{-C P(}(\widehat{\underline{a}})}{\rightleftarrows} \\
& \quad \stackrel{(\text { logic })}{\forall} \\
& \forall x_{1} \exists x_{2} \forall \tilde{x}_{1} \leq x_{1} \exists \widehat{x}_{2} \leq x_{2} \forall x_{3} \ldots \exists^{(d)} x_{k+1} \exists \tilde{x}_{2} \leq \widehat{x}_{2} \ldots \exists^{(d)} \tilde{x}_{k+1} \leq x_{k+1} A_{0}(\underline{a}, \underline{\tilde{x}}) \\
& \leftrightarrow \forall x_{1} \exists x_{2} \forall \tilde{x}_{1} \leq x_{1} \forall x_{3} \ldots \exists^{(d)} x_{k+1} \exists \tilde{x}_{2} \leq x_{2} \ldots \exists^{(d)} \tilde{x}_{k+1} \leq x_{k+1} A_{0}(\underline{a}, \underline{\tilde{x}}) \\
& \leftrightarrow \forall x_{1} \exists x_{2} \forall x_{3} \forall \tilde{x}_{1} \leq x_{1} \exists x_{4} \ldots \exists^{(d)} x_{k+1} \exists \tilde{x}_{2} \leq x_{2} \ldots \exists^{(d)} \tilde{x}_{k+1} \leq x_{k+1} A_{0}(\underline{a}, \underline{\tilde{x}}) .
\end{aligned}
$$

In the same way as we shifted $\forall \tilde{x}_{1} \leq x_{1}$ over $\exists x_{2}$ we now move $\forall \tilde{x}_{1} \leq x_{1}$ over $\exists x_{4}$, then permute $\forall \tilde{x}_{1} \leq x_{1}$ with $\forall x_{5}$, move over $\exists x_{6}$ and so on until we obtain $\tilde{A}(\underline{a})$. This requires only $\Pi_{k-3}^{0}$-instances (or simpler ones) of $\mathrm{CP}$ 
which can be considered a fortiori as instances $\Pi_{k-2}^{0} \mathrm{CP}\left(\zeta_{j} \underline{a}\right)$. Putting things together we have shown that (relative to $G_{n} A^{\omega}$ ):

$$
\Pi_{k-1^{-}}^{0} \mathrm{CP}(\widehat{\xi} \underline{a}) \wedge \bigwedge_{i} \Pi_{k-2^{-}}^{0} \mathrm{CP}\left(\xi_{i} \underline{a}\right) \wedge \bigwedge_{j} \Pi_{k-2^{-}}^{0} \mathrm{CP}\left(\zeta_{j} \underline{a}\right) \rightarrow(A(\underline{a}) \rightarrow \tilde{A}(\underline{a}))
$$

and

$$
\bigwedge_{i} \Pi_{k-2}^{0}-\mathrm{CP}\left(\xi_{i} \underline{a}\right) \wedge \bigwedge_{j} \Pi_{k-2}^{0}-\mathrm{CP}\left(\zeta_{j} \underline{a}\right) \rightarrow(\tilde{A}(\underline{a}) \rightarrow A(\underline{a}))
$$

which concludes the proof of the lemma.

Since in our main results we assume $n \geq 2$ or $n \geq 3$ for the level $n$ of $\mathrm{G}_{n} \mathrm{~A}^{\omega}$ we also use for simplicity $\mathrm{G}_{2} \mathrm{~A}^{\omega}$ in the following definition and lemmas although some of them can be carried out even in $\mathrm{G}_{1} \mathrm{~A}^{\omega}$.

Definition 3.11 (and lemma) For $m \in \mathbb{N}$ let $\Phi \in G_{2} R^{\omega}$ be such that

$$
\begin{aligned}
& G_{2} A^{\omega} \vdash \forall f^{(0) \ldots(0)}, x^{0}, y_{1}^{0}, z_{1}^{0}, \ldots, y_{m}^{0}, z_{m}^{0},\left(y_{m+1}\right) \\
&\left(\Phi f x y_{1} z_{1} \ldots y_{m} z_{m}\left(y_{m+1}\right)={ }_{0} 0\right. \\
& \leftrightarrow \forall \tilde{y}_{1} \leq y_{1} \exists \tilde{z}_{1} \leq z_{1} \ldots \forall \tilde{y}_{m} \leq y_{m} \exists \tilde{z}_{m} \leq z_{m}\left(\forall \tilde{y}_{m+1} \leq y_{m+1}\right) \\
&\left.\quad\left(f\left(x, \tilde{y}_{1}, \tilde{z}_{1}, \ldots, \tilde{y}_{m}, \tilde{z}_{m},\left(\tilde{y}_{m+1}\right)\right)={ }_{0} 0\right)\right) .
\end{aligned}
$$

We denote $\Phi f$ by $f^{\prime}$.

Lemma 3.12 Let $k \geq 1$. There are (effectively) finitely many terms $\xi_{1}, \ldots, \xi_{l} \in G_{2} R^{\omega}$ such that

$$
G_{2} A^{\omega} \vdash \forall f\left(\left(\bigwedge_{i=1}^{l} \Pi_{k-2}^{0}-C P\left(\xi_{i} f\right)\right) \rightarrow\left(\Pi_{k}^{0}-C A(f) \leftrightarrow \Pi_{k}^{0}-C A\left(f^{\prime}\right)\right)\right) .
$$

Proof: The lemma follows from lemma 3.10.

Definition 3.13 The 'monotone' tertium-non-datur is given by

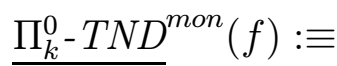

$$
\begin{aligned}
& \left\{\begin{array}{l}
\forall x^{0} \exists u_{1}^{0} \forall y_{1}^{0} \exists z_{1}^{0} \forall v_{1}^{0} \ldots \exists u_{m}^{0} \forall y_{m}^{0} \exists z_{m}^{0} \forall v_{m}^{0}\left(\exists u_{m+1}^{0} \forall y_{m+1}^{0}\right) \forall \tilde{x} \leq x \\
\left(f^{\prime}\left(\tilde{x}, y_{1}, z_{1}, \ldots, y_{m}, z_{m},\left(y_{m+1}\right)\right)={ }_{0} 0 \vee f^{\prime}\left(\tilde{x}, u_{1}, v_{1}, \ldots, u_{m}, v_{m},\left(u_{m+1}\right)\right) \neq 0\right),
\end{array}\right.
\end{aligned}
$$

Lemma 3.14 1) $G_{2} A^{\omega} \vdash \forall f\left(\left(\Pi_{k}^{0}-T N D^{\text {mon }}(f)\right)^{S} \rightarrow\left(\Pi_{k}^{0}-T N D\left(f^{\prime}\right)^{p r}\right)^{S}\right)$. 
2) $G_{2} A^{\omega} \vdash \forall f\left(M o n^{*}\left(\Pi_{k}^{0}-T N D^{m o n}(f)\right)\right)$.

Proof: 1) follows by putting $\tilde{x}:=x$.

2) Follows immediately from the definition of $\Pi_{k}^{0}$-TND ${ }^{\text {mon }}(f)$.

Proposition $3.15 G_{2} A^{\omega} \vdash \forall f\left(\left(\Pi_{k}^{0}-T N D^{m o n}(f)\right)^{S} \rightarrow \Pi_{k}^{0}-C A\left(f^{\prime}\right)\right)$.

Proof: Lemmas 3.5 and 3.14.1.

Lemma 3.16 One can construct $a \xi \in G_{2} R^{\omega}$ such that

$$
G_{2} A^{\omega}+A C^{0,0}-q f \vdash \forall f\left(\Pi_{k}^{0}-C A(\xi f) \rightarrow \Pi_{k}^{0}-C P(f)\right) .
$$

\section{Proof:}

Using $\Pi_{k}^{0}$ - $\mathrm{CA}(\xi f)$ for a suitable $\xi \in \mathrm{G}_{2} \mathrm{R}^{\omega}$ one can reduce $\Pi_{k}^{0}$ - $\mathrm{CP}(f)$ to $\Pi_{0}^{0}$-CP which is provable in $\mathrm{G}_{2} \mathrm{~A}^{\omega}+\mathrm{AC}^{0,0}$-qf.

Proposition 3.17 For a suitable $\xi \in G_{2} R^{\omega}$ one has

$$
G_{2} A^{\omega}+A C^{0,0}-q f \vdash \forall f\left(\left(\Pi_{k}^{0}-T N D^{m o n}(\xi f)\right)^{S} \rightarrow \Pi_{k}^{0}-C A(f)\right) .
$$

Proof: Induction on $k: k=0,1$ : easy. Let $k>1$ and lets assume that the proposition holds for all $m<k . \Pi_{k-2}^{0}-\mathrm{CP}\left(\xi_{i} f\right)$ denote the instances of $\Pi_{k-2}^{0}$-collection from lemma 3.12 which are needed to show

$$
\Pi_{k}^{0}-\mathrm{CA}(f) \leftrightarrow \Pi_{k}^{0}-\mathrm{CA}\left(f^{\prime}\right)
$$

Let $\widehat{\xi} \in \mathrm{G}_{2} \mathrm{R}^{\omega}$ be (using lemma 3.16) such that ${ }^{13}$

$$
(1) \mathrm{G}_{2} \mathrm{~A}^{\omega}+\mathrm{AC}^{0,0}-\mathrm{qf} \vdash \Pi_{k-2}^{0} \mathrm{CA}(\widehat{\xi} f) \rightarrow\left(\Pi_{k}^{0}-\mathrm{CA}(f) \leftrightarrow \Pi_{k}^{0}-\mathrm{CA}\left(f^{\prime}\right)\right) .
$$

By the induction hypothesis we have

$$
\text { (2) } \mathrm{G}_{2} \mathrm{~A}^{\omega}+\mathrm{AC}^{0,0} \text {-qf } \vdash \forall f\left(\left(\Pi_{k-2}^{0}-\mathrm{TND}^{m o n}(\tilde{\xi} f)\right)^{S} \rightarrow \Pi_{k-2}^{0}-\mathrm{CA}(f)\right)
$$

for a suitable $\tilde{\xi} \in \mathrm{G}_{2} \mathrm{R}^{\omega}$. So by proposition $3.15(3): \equiv$

$$
\mathrm{G}_{2} \mathrm{~A}^{\omega}+\mathrm{AC}^{0,0}-\mathrm{qf} \vdash\left(\Pi_{k}^{0}-\mathrm{TND}^{\text {mon }}(f)\right)^{S} \wedge\left(\Pi_{k-2}^{0} \mathrm{TND}^{\text {mon }}(\tilde{\xi}(\widehat{\xi} f))\right)^{S} \rightarrow \Pi_{k}^{0} \mathrm{CA}(f) .
$$

\footnotetext{
${ }^{13}$ Note that two instances $\Pi_{k}^{0}-\mathrm{CA}\left(\xi_{1} f\right) \wedge \Pi_{k}^{0}-\mathrm{CA}\left(\xi_{2} f\right)$ can be coded together into one instance $\Pi_{k}^{0}-\mathrm{CA}\left(\xi_{3} f\right)$ in $\mathrm{G}_{2} \mathrm{~A}^{\omega}$.
} 
Introducing dummy quantifiers, $\left(\Pi_{k-2}^{0}-\mathrm{TND}^{m o n}(\tilde{\xi}(\widehat{\xi} f))\right)^{S}$ can be reduced to $\left(\Pi_{k}^{0}-\mathrm{TND}^{\text {mon }}\left(\xi^{*} f\right)\right)^{S}$ for a suitable $\xi^{*} \in \mathrm{G}_{2} \mathrm{R}^{\omega}$. Furthermore

(4) $\left(\Pi_{k}^{0}-\mathrm{TND}^{\text {mon }}(h)\right)^{S} \rightarrow\left(\Pi_{k}^{0}-\mathrm{TND}^{\text {mon }}(f)\right)^{S} \wedge\left(\Pi_{k}^{0}-\mathrm{TND}^{\text {mon }}(g)\right)^{S}$

for

$$
h(x, \underline{y}, \underline{z})=\left\{\begin{array}{l}
f(\tilde{x}, \underline{y}, \underline{z}) \text { if } x=2 \tilde{x} \\
g(\tilde{x}, \underline{y}, \underline{z}) \text { if } x=2 \tilde{x}+1 .
\end{array}\right.
$$

Hence

(5) $\left(\Pi_{k}^{0}-\mathrm{TND}^{\text {mon }}(\xi f)\right)^{S} \rightarrow\left(\Pi_{k}^{0}-\mathrm{TND}^{\text {mon }}(f)\right)^{S} \wedge\left(\Pi_{k}^{0}-\mathrm{TND}^{\text {mon }}\left(\xi^{*} f\right)\right)^{S}$

for a suitable $\xi \in \mathrm{G}_{2} \mathrm{R}^{\omega}$. By (3) and (5) we have

$$
\mathrm{G}_{2} \mathrm{~A}^{\omega}+\mathrm{AC}^{0,0}-\mathrm{qf} \vdash\left(\Pi_{k}^{0}-\mathrm{TND}^{m o n}(\xi f)\right)^{S} \rightarrow \Pi_{k}^{0}-\mathrm{CA}(f) .
$$

Lemma 3.18 Let $k \geq 1$ and $A \in \Sigma_{k-1}^{0}$. Then

$$
G_{3} A^{\omega}+\Sigma_{k}^{0}-I A \vdash \forall x^{0} \exists u^{0} \forall \tilde{x} \leq_{0} x\left(\forall y^{0} A(\tilde{x}, y) \vee \exists \tilde{u} \leq u \neg A(\tilde{x}, \tilde{u})\right) .
$$

Proof: Assume

$$
(+) \forall u^{0} \exists \tilde{x} \leq x(\exists y \neg A(\tilde{x}, y) \wedge \forall \tilde{u} \leq u A(\tilde{x}, \tilde{u})) .
$$

We show by induction on $n$ :

$$
(*) \forall n \exists u, \tilde{x} \overbrace{\left(\begin{array}{l}
l t h \tilde{x}=n+1 \wedge \bigwedge_{\substack{i, j \leq n \\
i \neq j}}\left((\tilde{x})_{i} \neq(\tilde{x})_{j} \wedge\right. \\
\bigwedge_{i \leq n}\left((\tilde{x})_{i} \leq x\right) \wedge \forall i \leq n \exists \tilde{u} \leq u \neg A\left((\tilde{x})_{i}, \tilde{u}\right)
\end{array}\right)}^{G(n, u, \tilde{x})}
$$

(For $n=x+1$ this obviously is contradictory and so $\neg(+)$ is proved).

$n=0:(+)$ applied to $u:=0$ yields an $x_{0} \leq x$ such that $A\left(x_{0}, 0\right)$ and $\exists y_{0} \neg A\left(x_{0}, y_{0}\right) .(*)$ is now satisfied by taking $\tilde{x}:=\left\langle x_{0}\right\rangle, u:=y_{0}$.

$n \rightarrow n+1$ : Let $u, \tilde{x}$ be such that $(*)$ is satisfied for $n$. By $(+)$ there exists an $x_{n+1} \leq x$ such that $\exists y_{n+1} \neg A\left(x_{n+1}, y_{n+1}\right)$ and $\forall \tilde{u} \leq u A\left(x_{n+1}, \tilde{u}\right)$. By $(*)$ we have $\forall i \leq n \exists \tilde{u} \leq u \neg A\left((\tilde{x})_{i}, \tilde{u}\right)$. Hence $\forall i \leq n\left((\tilde{x})_{i} \neq x_{n+1}\right)$ and so $\widehat{u}:=\max \left(u, y_{n+1}\right), \widehat{x}:=\tilde{x} *\left\langle x_{n+1}\right\rangle$ satisfy $G(n+1, \widehat{u}, \widehat{x})$.

It remains to show that $\exists u, \tilde{x} G(n, u, \tilde{x})$ is equivalent to a $\Sigma_{k}^{0}$-formula: 
Using $\Sigma_{k-1}^{0}$ CP,$\exists \tilde{u} \leq u \neg A\left((\tilde{x})_{i}, \tilde{u}\right)$ can be shown to be equivalent to a $\Pi_{k-1^{-}}^{0}$ formula. Since $\Sigma_{k-1}^{0}$-CP follows from $\Sigma_{k}^{0}$-IA, the whole proof can be carried out in $\mathrm{G}_{3} \mathrm{~A}^{\omega}+\Sigma_{k}^{0}$-IA.

In contrast to $\Pi_{k}^{0}$-TND $(f)$ its monotone version $\Pi_{k}^{0}$-TND ${ }^{\text {mon }}(f)$ does not hold logically. However it can be proved using $\Sigma_{k}^{0}$-induction. More precisely the following proposition holds:

Proposition 3.19 Let $k \geq 1$. There are finitely many instances $\Sigma_{k}^{0}-I A\left(\xi_{i} f\right)$ such that

$$
G_{3} A^{\omega} \vdash \forall f\left(\left(\bigwedge_{i=1}^{l} \Sigma_{k}^{0}-I A\left(\xi_{i} f\right)\right) \rightarrow \Pi_{k}^{0}-T N D^{m o n}(f)\right)
$$

Proof: By (the proof of) lemma 3.18 there are instances $\Sigma_{k}^{0} \mathrm{IA}\left(\xi_{i} f\right)$ which prove (relatively to $\mathrm{G}_{3} \mathrm{~A}^{\omega}$ )

$(*)\left\{\begin{array}{c}\forall x \exists u_{1} \forall \tilde{x} \leq x\left(\forall y_{1} \exists z_{1} \ldots \forall y_{m} \exists z_{m}\left(\forall y_{m+1}\right)\left(f^{\prime}\left(\tilde{x}, y_{1}, z_{1}, \ldots, y_{m}, z_{m}\right)=0\right)\right. \\ \left.\vee \exists \tilde{u} \leq u_{1} \forall v_{1} \ldots \exists u_{m} \forall v_{m}\left(\exists u_{m+1}\right)\left(f^{\prime}\left(\tilde{x}, \tilde{u}, v_{1}, \ldots, u_{m}, v_{m}\right) \neq 0\right)\right)\end{array}\right.$

and therefore by the definition of $f^{\prime}$ (which makes

$\exists \tilde{u} \leq u_{1} \forall v_{1} \ldots \exists u_{m} \forall v_{m}\left(\exists u_{m+1}\right)\left(f^{\prime}\left(\tilde{x}, \tilde{u}, v_{1}, \ldots, u_{m}, v_{m},\left(u_{m+1}\right)\right) \neq 0\right)$ monotone w.r.t. $\exists \tilde{u})$

$$
\left\{\begin{array}{c}
\forall x \exists u_{1} \forall \tilde{x} \leq x\left(\forall y_{1} \exists z_{1} \ldots \forall y_{m} \exists z_{m}\left(\forall y_{m+1}\right)\left(f^{\prime}\left(\tilde{x}, y_{1}, z_{1}, \ldots, y_{m}, z_{m},\right)=0\right)\right. \\
\left.\vee \forall v_{1} \ldots \exists u_{m} \forall v_{m}\left(\exists u_{m+1}\right)\left(f^{\prime}\left(\tilde{x}, u_{1}, v_{1}, \ldots, u_{m}, v_{m},\right) \neq 0\right)\right),
\end{array}\right.
$$

which is equivalent to $(* *): \equiv$

$$
\left\{\begin{array}{c}
\forall x \exists u_{1} \forall y_{1} \forall \tilde{x} \leq x \exists z_{1}\left(\forall y_{2} \ldots \forall y_{m} \exists z_{m}\left(\forall y_{m+1}\right)\left(f^{\prime}\left(\tilde{x}, y_{1}, z_{1}, \ldots, y_{m}, z_{m},\right)=0\right)\right. \\
\left.\vee \forall v_{1} \ldots \exists u_{m} \forall v_{m}\left(\exists u_{m+1}\right)\left(f^{\prime}\left(\tilde{x}, u_{1}, v_{1}, \ldots, u_{m}, v_{m},\right) \neq 0\right)\right) .
\end{array}\right.
$$

By a suitable instance of $\Pi_{k-1}^{0}$-CP and the monotonicity of (**) w.r.t. $\exists z_{1}$ one can 'shift' $\forall \tilde{x} \leq x$ over $\exists z_{1}$. Now one continues in this way until one obtains $\Pi_{k}^{0}-\mathrm{TND}^{\text {mon }}(f)$ which needs only suitable instances of $\Pi_{l}^{0}$-CP with $l<k-1$ which can be considered as instances of $\Pi_{k-1}^{0}$ CP. All the instances of $\Pi_{k-1}^{0}$-CP used follow from suitable instances of $\Sigma_{k}^{0}$-IA.

Corollary 3.20 $G_{3} A^{\omega} \vdash \forall f\left(\Pi_{k}^{0}-C A(\xi f) \rightarrow \Pi_{k}^{0} T N D^{\text {mon }}(f)\right)$ for a suitable $\xi \in G_{3} R^{\omega}$. 


\section{Conservation results for $\prod_{k}^{0}-\mathrm{AC}(f)$ and $\Delta_{k}^{0}$ - $\mathbf{C A}(f, g)$}

We are now ready to determine the arithmetical content of instances $\Pi_{k}^{0}$ $\mathrm{CA}(\xi u v)$ and even $\Pi_{k}^{0}-\mathrm{AC}(\xi u v)$ and $\Delta_{k+1}^{0}-\mathrm{CA}(\xi u v)$ in proofs of monotone sentences (and without monotonicity assumption if the logical complexity is restricted). It turns out that this content is given by certain instances of $\Pi_{k}^{0}$-TND ${ }^{\text {mon }}$.

\section{Definition 4.1}

$$
\begin{aligned}
& \Pi_{k}^{0}-A C(f): \equiv\left\{\begin{aligned}
\forall l^{0}\left(\forall x^{0} \exists y^{0} \forall u_{1}^{0} \exists u_{2}^{0} \ldots \exists^{(d)} u_{k}^{0}\left(f(l, x, y, \underline{u})={ }_{0} 0\right)\right. \\
\left.\rightarrow \exists g^{1} \forall x^{0} \forall u_{1}^{0} \exists u_{2}^{0} \ldots \exists^{(d)} u_{k}^{0}\left(f(l, x, g x, \underline{u})={ }_{0} 0\right)\right)
\end{aligned}\right. \\
& \Delta_{k}^{0}-C A(f, g): \equiv \\
& \left\{\begin{aligned}
\forall l^{0} & \left(\forall x ^ { 0 } \left(\left[\forall u_{1}^{0} \exists u_{2}^{0} \ldots \exists^{(d)} u_{k}^{0}\left(f(l, x, \underline{u})={ }_{0} 0\right) \leftrightarrow\right.\right.\right. \\
& \left.\left.\exists v_{1}^{0} \forall v_{2}^{0} \ldots \forall^{(d)} v_{k}^{0}\left(g(l, x, \underline{v})={ }_{0} 0\right)\right]\right) \\
\rightarrow & \left.\exists h^{1} \forall x^{0}\left(g x={ }_{0} 0 \leftrightarrow \forall u_{1} \exists u_{2} \ldots \exists^{(d)} u_{k}\left(f(l, x, \underline{u})={ }_{0} 0\right)\right)\right)
\end{aligned}\right.
\end{aligned}
$$

$\Delta_{k}^{0}-C A(f): \equiv \Delta_{k}^{0}-C A\left(j_{1}^{1} f, j_{2}^{1} f\right)$ for the projection functions $j_{i}^{1} \in G_{2} R^{\omega}$.

Lemma 4.2 Let $k \in \mathbb{N}$. Then for suitable $\xi_{1}, \xi_{2} \in G_{2} R^{\omega}$ :

1) $G_{2} A^{\omega}+A C^{0,0}-q f \vdash \forall f\left(\Pi_{k}^{0}-C A\left(\xi_{1} f\right) \rightarrow \Pi_{k}^{0}-A C(f)\right)$.

2) $G_{2} A^{\omega}+A C^{0,0}-q f \vdash \forall f\left(\Pi_{k}^{0}-C A\left(\xi_{2} f\right) \rightarrow \Delta_{k+1}^{0}-C A(f)\right)$.

\section{Proof: Obvious.}

Below we also need a certain 'non-standard' axiom $F^{-}$

$$
\begin{aligned}
& F^{-}: \equiv \\
& \forall \Phi^{2(0)}, y^{1(0)} \exists y_{0} \leq_{1(0)} y \forall k^{0}, z^{1}, n^{0}\left(\bigwedge_{i<0_{0} n}\left(z i \leq_{0} y k i\right) \rightarrow \Phi k(\overline{z, n}) \leq_{0} \Phi k\left(y_{0} k\right)\right),
\end{aligned}
$$

where, for $z^{\rho 0},(\overline{z, n})\left(k^{0}\right):={ }_{\rho} z k$, if $k<_{0} n$ and $:=0^{\rho}$, otherwise.

$F^{-}$does not hold in the full set-theoretic type-structure but can be eliminated from proofs of monotone sentences in our theories. This axiom was introduced and studied in [12] and implies the principle of uniform $\Sigma_{1}^{0}$ boundedness which was mentioned in the introduction and which will be generalized in section 5 below. 
Proposition 4.3 Let $n \geq 2, k \geq 0$ and

$B: \equiv \forall u^{1} \forall v \leq_{\tau} t u \exists a_{1}^{0} \forall b_{1}^{0} \ldots \exists a_{l}^{0} \forall b_{l}^{0} \exists w^{\gamma} B_{0}$ be a sentence in $\mathcal{L}\left(G_{n} A^{\omega}\right)$, where $B_{0}$ is quantifier-free and $t \in G_{n} R^{\omega}$. Let $\xi_{1}, \xi_{2} \in G_{n} R^{\omega}$ (of suitable types) and $\Delta$ a set of sentences having the form $\forall x^{\delta} \exists y \leq_{\rho} s x \forall z^{\eta} A_{0}$ ( $A_{0}$ quantifier-free, $\left.s \in G_{n} R^{\omega}\right)$. Then for a suitable $\xi \in G_{n} R^{\omega}$ the following holds:

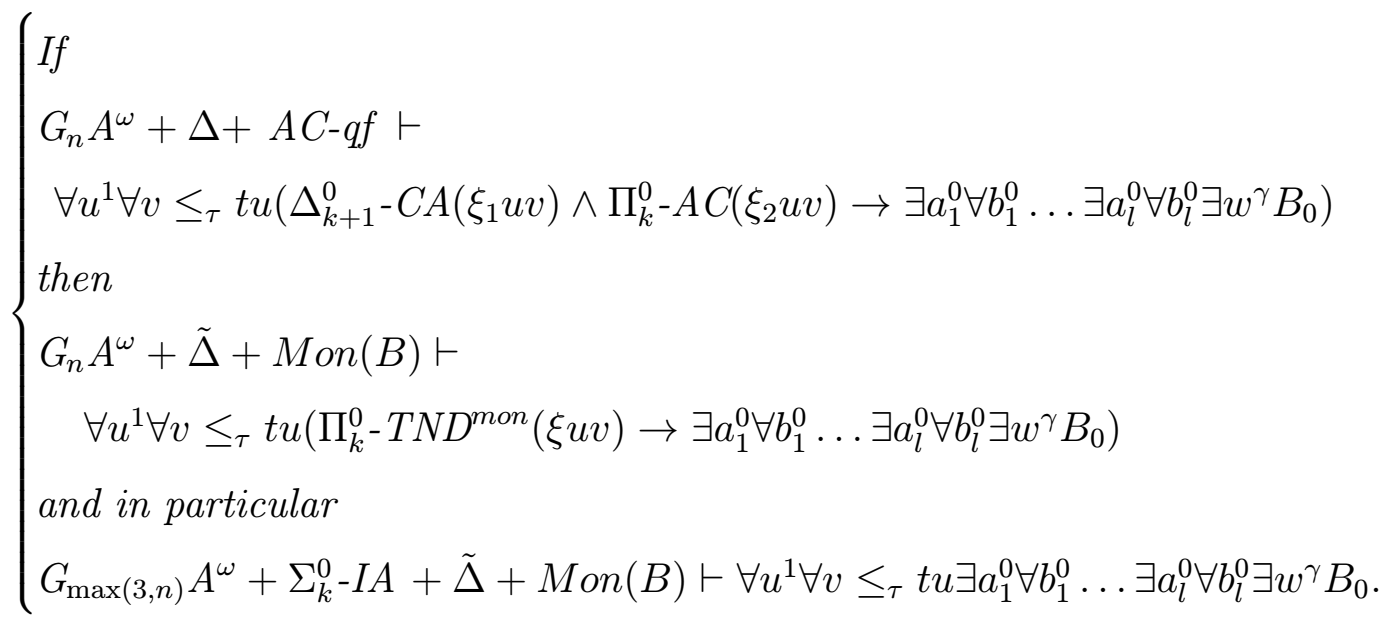

In the assumption of the rule the theory $G_{n} A^{\omega}+\Delta+A C$-qf can be strengthened ${ }_{t o}{ }^{14}\left(G_{n} A^{\omega}+\Delta+A C-q f\right) \oplus F^{-}$. Then in the first conclusion $G_{n} A^{\omega}$ must be replaced by $G_{\max (3, n)} A^{\omega}$.

Proof: By lemma 4.2, proposition 3.17 and the fact that two instances of $\Pi_{k}^{0}$-CA can be coded together into a single instance of $\Pi_{k}^{0}$-CA, there is a $\xi \in$ $\mathrm{G}_{n} \mathrm{R}^{\omega}$ such that

$$
\begin{aligned}
& \mathrm{G}_{n} \mathrm{~A}^{\omega}+\mathrm{AC}^{0,0}-\mathrm{qf} \\
& \vdash \forall u^{1} \forall v \leq_{\tau} t u\left(\left(\Pi_{k}^{0}-\mathrm{TND}^{m o n}(\xi u v)\right)^{S} \rightarrow \Delta_{k+1}^{0} \mathrm{CA}\left(\xi_{1} u v\right) \wedge \Pi_{k}^{0}-\mathrm{AC}\left(\xi_{2} u v\right) .\right.
\end{aligned}
$$

So the assumption of the rule implies

$$
\left\{\begin{array}{l}
\mathrm{G}_{n} \mathrm{~A}^{\omega}+\mathrm{AC}-\mathrm{qf}+\Delta \vdash \\
\forall u^{1} \forall v \leq_{\tau} t u\left(\Pi_{k}^{0}-\mathrm{TND}^{m o n}(\xi u v) \rightarrow \exists a_{1}^{0} \forall b_{1}^{0} \ldots \exists a_{l}^{0} \forall b_{l}^{0} \exists w^{\gamma} B_{0}\right) .
\end{array}\right.
$$

By lemma 3.14.2) the prenexation ${ }^{15}$

$$
A^{p r}: \equiv \forall u^{1} \forall v \leq_{\tau} t u \exists x \forall u_{1} \exists y_{1} \forall z_{1} \exists v_{1} \ldots \exists a_{1} \forall b_{1} \ldots \exists w^{\gamma}\left(\mathrm{TND}_{0}^{\text {mon }}(\xi u v) \rightarrow B_{0}\right)
$$

\footnotetext{
${ }^{14}$ Here $\oplus$ means that $F^{-}$must not be used in the proof of the premise of an application of the quantifier-free rule of extensionality $\mathrm{QF}-\mathrm{ER} . \mathrm{G}_{n} \mathrm{~A}^{\omega}$ satisfies the deduction theorem w.r.t $\oplus$ but not w.r.t + .

${ }^{15}$ Note that $A^{p r}$ is not completely in prenex normal form because of the universal quantifiers hidden in $v \leq_{\tau} t u$. However it has

the form required in theorem 2.7 used below.
} 
of $^{16}$

$$
A: \equiv \forall u^{1} \forall v \leq_{\tau} t u\left(\Pi_{k}^{0}-\operatorname{TND}^{m o n}(\xi u v) \rightarrow \exists a_{1}^{0} \forall b_{1}^{0} \ldots \exists a_{l}^{0} \forall b_{l}^{0} \exists w^{\gamma} B_{0}\right)
$$

is monotone if $B$ is:

$$
\mathrm{G}_{n} \mathrm{~A}^{\omega} \vdash \operatorname{Mon}(B) \rightarrow \operatorname{Mon}\left(A^{p r}\right) .
$$

Now (1) implies

$$
\mathrm{G}_{n} \mathrm{~A}^{\omega}+\mathrm{AC}-\mathrm{qf}+\Delta \vdash\left(A^{p r}\right)^{H}
$$

and therefore using theorem 2.7

$$
\begin{gathered}
\mathrm{G}_{n} \mathrm{~A}^{\omega}+\tilde{\Delta}+\operatorname{Mon}(B) \vdash A^{p r} \text { i.e. } \\
\mathrm{G}_{n} \mathrm{~A}^{\omega}+\tilde{\Delta}+\operatorname{Mon}(B) \vdash A .
\end{gathered}
$$

The second part of the claim in the proposition now follows from proposition 3.19 .

The proof above can be combined with the elimination procedure for $F^{-}$ given in [12](thm.4.21) yielding the claim about adding $F^{-}$.

Corollary 4.4 Let $k \geq 1, \gamma \leq 2$ and $\xi_{1}, \xi_{2} \in G_{n} R^{\omega}$. Then the following rule holds

$$
\left\{\begin{array}{l}
G_{\infty} A^{\omega}+\Delta+A C-q f \vdash \\
\quad \forall u^{1} \forall v \leq_{\tau} t u\left(\Delta_{k+1}^{0}-C A\left(\xi_{1} u v\right) \wedge \Pi_{k}^{0}-A C\left(\xi_{2} u v\right) \rightarrow \exists w^{\gamma} B_{0}(u, v, w)\right) \\
\Rightarrow \exists \Phi \in T_{k-1} \text { such that } \\
\mathrm{PA}_{i}^{\omega}+\tilde{\Delta} \vdash \forall u^{1} \forall v \leq_{\tau} t u \exists w \leq_{\gamma} \Phi u B_{0}(u, v, w) .
\end{array}\right.
$$

Again we may strengthen the theory in the assumption of the rule above by $\oplus F^{-}$.

Proof: The corollary follows from proposition 4.3 by observing that the condition $\operatorname{Mon}\left(\forall u^{1} \forall v \leq_{\tau} t u \exists w^{\gamma} B_{0}\right)$ is empty and using the fact that $\mathrm{G}_{\infty} \mathrm{A}^{\omega}+$ $\tilde{\Delta}+\Sigma_{k}^{0}$-IA has a monotone functional interpretation as developed in [9] (via negative translation) in $\mathrm{PA}_{i}^{\omega}+\tilde{\Delta}$ by terms $\in T_{k-1}$. The latter follows from the proof that the negative translation of $\Sigma_{k}^{0}$-IA has a functional interpretation in $T_{k-1}$ (provable in (a subsystem of) $\mathrm{PA}_{i}^{\omega}$ ) as given in [19] and the fact that every (closed) term of $T_{k-1}$ can be majorized (in the sense of definition 2.1) by a suitable term in $T_{k-1}$ which follows from Howard's proof of this fact for full $T$ as given in [6].

\footnotetext{
${ }^{16} \mathrm{TND}_{0}^{\text {mon }}$ denotes the quantifier-free matrix of (some prenex normal form of) $\Pi_{k}^{0}$ $\mathrm{TND}^{\text {mon }}$.
} 
Corollary 4.5 Let $n \geq 3$ and $A$ be a $\Pi_{1}^{1}$-sentence.

$$
\begin{aligned}
& \text { If } E-G_{n} A^{\omega}+A C^{1,0}-q f+\Delta_{k+1}^{0}-C A^{-}+\Pi_{k}^{0}-A C^{-}+W K L \vdash A \\
& \text { then } G_{n} A^{\omega}+\Sigma_{k}^{0}-I A+\operatorname{Mon}(A) \vdash A \text {. }
\end{aligned}
$$

\section{Proof:}

Using the deduction theorem for $E-G_{n} A^{\omega}$, the fact that $E-G_{3} A^{\omega}+A C^{1,0}-q f$ $+F^{-}$proves WKL (see [12]) and the existence of characteristic terms $\in \mathrm{G}_{n} \mathrm{R}^{\omega}$ for quantifier-free formulas of $\mathrm{E}-\mathrm{G}_{n} \mathrm{~A}^{\omega}$ the assumption implies

$$
\mathrm{E}^{-\mathrm{G}_{n}} \mathrm{~A}^{\omega}+\mathrm{AC}^{1,0}-\mathrm{qf}+F^{-} \vdash \bigwedge_{i=1}^{l}\left(\Delta_{k+1}^{0}-\mathrm{CA}\left(\xi_{i}\right)\right) \wedge \bigwedge_{j=1}^{\tilde{l}}\left(\Pi_{k}^{0}-\mathrm{AC}\left(\tilde{\xi}_{j}\right)\right) \rightarrow A
$$

for certain terms $\xi_{i}, \tilde{\xi}_{j} \in \mathrm{G}_{n} \mathrm{R}^{\omega}$ (corresponding to the universal closures of the instances of $\Delta_{k+1}^{0}-\mathrm{CA}^{-}$and $\Pi_{k}^{0}-\mathrm{AC}^{-}$used in the proof).

For suitable $\xi, \tilde{\xi} \in \mathrm{G}_{n} \mathrm{R}^{\omega}$ we have

$$
\mathrm{G}_{n} \mathrm{~A}^{\omega} \vdash \Delta_{k+1}^{0} \mathrm{CA}(\xi) \rightarrow \bigwedge_{i=1}^{l}\left(\Delta_{k+1}^{0}-\mathrm{CA}\left(\xi_{i}\right)\right)
$$

and

$$
\mathrm{G}_{n} \mathrm{~A}^{\omega} \vdash \Pi_{k}^{0}-\mathrm{AC}(\tilde{\xi}) \rightarrow \bigwedge_{j=1}^{\tilde{l}}\left(\Pi_{k}^{0}-\mathrm{AC}\left(\tilde{\xi}_{j}\right)\right) .
$$

Together with elimination of extensionality (see e.g. [16]) we obtain

$$
\left(\mathrm{G}_{n} \mathrm{~A}^{\omega}+\mathrm{AC}^{1,0}-\mathrm{qf}\right) \oplus F^{-} \vdash \Delta_{k+1}^{0} \mathrm{CA}(\xi) \wedge \Pi_{k}^{0}-\mathrm{AC}(\tilde{\xi}) \rightarrow A .
$$

The conclusion now follows from proposition 4.3.

Lemma 4.6 Let $\forall u^{1} \forall v \leq_{\tau}$ tu $A(u, v)$ be a sentence with $A(u, v) \in \Sigma_{k+1}^{0}$. Then one can construct a sentence $\forall u^{1} \forall v \leq_{\tau}$ tu $\tilde{A}(u, v)$ with $\tilde{A}(u, v) \in \Sigma_{k+1}^{0}$ such that

1) $G_{n} A^{\omega} \vdash \operatorname{Mon}\left(\forall u^{1} \forall v \leq_{\tau} t u \tilde{A}(u, v)\right)$,

2) $G_{n} A^{\omega} \vdash \forall u^{1} \forall v \leq_{\tau} t u\left(\bigwedge_{i=1}^{l} \Pi_{k-2}^{0}-C P\left(\xi_{i} u v\right) \rightarrow(A(u, v) \rightarrow \tilde{A}(u, v))\right)$,

3) $G_{n} A^{\omega} \vdash \forall u^{1} \forall v \leq_{\tau} t u\left(\bigwedge_{i=1}^{\tilde{l}} \Pi_{k-1}^{0}-C P\left(\tilde{\xi}_{i} u v\right) \rightarrow(\tilde{A}(u, v) \rightarrow A(u, v))\right)$, 
where $\xi_{i}, \tilde{\xi}_{j} \in G_{n} R^{\omega}$ are suitable terms.

Proof: Lemmas 3.8,3.10.

Corollary 4.7 Let $n \geq 3, \forall u^{1} \forall v \leq_{\tau}$ tu $A(u, v)$ be a sentence in $G_{n} A^{\omega}$ with $A(u, v) \in \Sigma_{k+1}^{0}$,

$t \in G_{n} R^{\omega}$ and $\xi_{1}, \xi_{2} \in G_{n} R^{\omega}$ of suitable types. Then the following rule holds:

$$
\left\{\begin{array}{l}
\text { If } G_{n} A^{\omega}+\Delta+A C-q f \vdash \\
\quad \forall u^{1} \forall v \leq_{\tau} t u\left(\Delta_{k+1}^{0}-C A\left(\xi_{1} u v\right) \wedge \Pi_{k}^{0}-A C\left(\xi_{2} u v\right) \rightarrow A(u, v)\right) \\
\text { then } G_{n} A^{\omega}+\Sigma_{k}^{0}-I A+\tilde{\Delta} \vdash \forall u^{1} \forall v \leq_{\tau} \text { tu } A(u, v) .
\end{array}\right.
$$

We may strengthen the theory in the assumption of the rule above by $\oplus F^{-}$.

\section{Proof:}

Let $\tilde{A}$ be as in lemma 4.6. $\Pi_{k-2}^{0}-\mathrm{CP}\left(\xi_{i} u v\right)$ follows from a corresponding instance $\Pi_{k-2}^{0}-\mathrm{AC}\left(\widehat{\xi}_{i} u v\right)$ of $\Pi_{k-2}^{0}$-AC which can be considered as an instance $\Pi_{k}^{0}-\mathrm{AC}\left(\widehat{\xi}_{i} u v\right)$ of $\Pi_{k}^{0}$-AC. All these instances $\Pi_{k}^{0}-\mathrm{AC}\left(\widehat{\xi}_{i} u v\right)(i=1, \ldots, l)$ can be combined with $\Pi_{k}^{0}-\mathrm{AC}\left(\xi_{2} u v\right)$ into a single instance $\Pi_{k}^{0}-\mathrm{AC}\left(\widehat{\xi}_{2} u v\right)$. Hence the assumption of the corollary yields

$$
\begin{aligned}
\mathrm{G}_{n} \mathrm{~A}^{\omega}+ & \Delta+\text { AC-qf } \vdash \\
& \forall u^{1} \forall v \leq_{\tau} t u\left(\Delta_{k+1}^{0}-\mathrm{CA}\left(\xi_{1} u v\right) \wedge \Pi_{k}^{0}-\mathrm{AC}\left(\widehat{\xi}_{2} u v\right) \rightarrow \tilde{A}(u, v)\right) .
\end{aligned}
$$

The conclusion now follows from proposition 4.3, lemma 4.6 and the fact that $\mathrm{G}_{n} \mathrm{~A}^{\omega}+\Sigma_{k}^{0} \mathrm{IA} \vdash \Pi_{k-1}^{0}-\mathrm{CP}$.

Corollary 4.8 For $n \geq 3$,

$E-G_{n} A^{\omega}+A C^{1,0}-q f+\Delta_{k+1}^{0}-C A^{-}+\Pi_{k}^{0}-A C^{-}+W K L$ is conservative w.r.t. $\Pi_{k+2}^{0}$-sentences over $G_{n} A^{\omega}+\Sigma_{k}^{0}-I A^{-}$.

Proof: The corollary follows from the proofs of corollary 4.5 and corollary 4.7 .

Remark 4.9 Corollary 4.8 is optimal in the following sense. For every $k$ there is a sentence $A \in \Pi_{k+3}^{0}$ such that

$$
\mathrm{G}_{3} \mathrm{~A}^{\omega}+\Pi_{k}^{0}-\mathrm{AC}^{-} \vdash A \text {, but } \mathrm{G}_{3} \mathrm{~A}^{\omega}+\Sigma_{k}^{0} \mathrm{IA} \forall \forall .
$$

Proof: There is a first-order instance $A$ (i.e. without parameters of type level $>0$ ) of $\Pi_{k}^{0}$-FAC which does not follow from $\Sigma_{k}^{0}$-IA relative to e.g. $\mathrm{G}_{3} \mathrm{~A}^{\omega}$ (see 
[18]). It is clear that $\mathrm{G}_{3} \mathrm{~A}^{\omega}+\Pi_{k}^{0}-\mathrm{AC}^{-} \vdash A$. Since the universal closure of $A$ can be shown to be equivalent to a $\Pi_{k+3}^{0}$-sentence in $\mathrm{G}_{3} \mathrm{~A}^{\omega}+\Sigma_{k}^{0}$-IA ${ }^{-}$(and hence in $\mathrm{G}_{3} \mathrm{~A}^{\omega}+\Pi_{k}^{0}-\mathrm{AC}^{-}$), the claim follows.

Corollary 4.10 Let $\forall u^{1} \forall v \leq_{\tau}$ tu $A(u, v)$ be a sentence with $A(u, v) \in \Sigma_{k+2}^{0}$. Then for $n \geq 3$ the following rule holds:

$$
\left\{\begin{array}{l}
\text { If } G_{n} A^{\omega}+\Delta+A C-q f \vdash \\
\quad \forall u^{1} \forall v \leq_{\tau} t u\left(\Delta_{k+1}^{0}-C A\left(\xi_{1} u v\right) \wedge \Pi_{k}^{0}-A C\left(\xi_{2} u v\right) \rightarrow A(u, v)\right) \\
\text { then } G_{n} A^{\omega}+\Pi_{k}^{0}-C P+\tilde{\Delta} \vdash \forall u^{1} \forall v \leq_{\tau} t u A(u, v) .
\end{array}\right.
$$

We may strengthen the theory in the assumption of the rule above by $\oplus F^{-}$.

Proof: The corollary follows analogously to the proof of corollary 4.7 using lemma 4.6 for $k+1$ instead of $k$ and the well-known fact (see e.g. [18]) that $\mathrm{G}_{n} \mathrm{~A}^{\omega}+\Pi_{k}^{0}$-CP $\vdash \Sigma_{k}^{0}$-IA.

Corollary 4.11 For $n \geq 3$, $E-G_{n} A^{\omega}+A C^{1,0}-q f+\Delta_{k+1}^{0}-C A^{-}+\Pi_{k}^{0}-A C^{-}+W K L$ is conservative w.r.t. $\Pi_{k+3}^{0}$-sentences over $G_{n} A^{\omega}+\Pi_{k}^{0}-C P^{-}$.

Proof: The corollary follows from corollary 4.10 analogously to the proof of corollary 4.8 .

Let EA be Kalmar-elementary arithmetic EA (with number quantifiers) and let us consider the variant $\mathrm{G}_{n} \mathrm{~A}_{-}^{\omega}$ of $\mathrm{G}_{n} \mathrm{~A}^{\omega}$ where the arbitrary true universal axioms 9) from its definition in [12] are replaced by the schema of quantifierfree induction (with arbitrary parameters) ${ }^{17}$ only. The results above also hold for $\mathrm{G}_{n} \mathrm{~A}_{-}^{\omega}$ since no other universal axioms from 9) were used. EA can be considered as a subsystem of $\mathrm{G}_{3} \mathrm{~A}_{-}^{\omega}$ and the latter is conservative over the former. Hence we obtain the following corollaries for EA:

Corollary 4.12 Let $A$ be an arbitrary sentence of EA. Then the following rule holds:

$$
\mathrm{EA}+\Pi_{k}^{0}-C P \vdash A \Rightarrow \mathrm{EA}+\Sigma_{k}^{0}-I A+\operatorname{Mon}(A) \vdash A .
$$

In particular we have the following

Corollary 4.13 Let $A, \tilde{A}$ be sentences from EA such that

\footnotetext{
${ }^{17}$ Or equivalently the second-order axiom of quantifier-free induction.
} 
1) $E A+\Pi_{k}^{0}-C P \vdash A \rightarrow \tilde{A}$,

2) $E A+\Sigma_{k}^{0}-I A \vdash \tilde{A} \rightarrow A$ and

3) $E A+\Sigma_{k}^{0}-I A \vdash \operatorname{Mon}(\tilde{A})$.

Then $E A+\Pi_{k}^{0}-C P \vdash A$ implies $E A+\Sigma_{k}^{0}-I A \vdash A$.

Combined with lemma 4.6 we finally obtain

Corollary 4.14 (Paris-Kirby [17], H. Friedman)

$E A+\Pi_{k}^{0}$-CP is $\Pi_{k+2}^{0}$-conservative over $E A+\Sigma_{k}^{0}-I A$.

\section{Generalized principles of uniform bound- edness and their arithmetical content}

In the following we define a generalization of the principle of uniform $\Sigma_{1}^{0}$ boundedness $\Sigma_{1}^{0}$ UB $^{-}$which was studied in [12],[14],[15]:

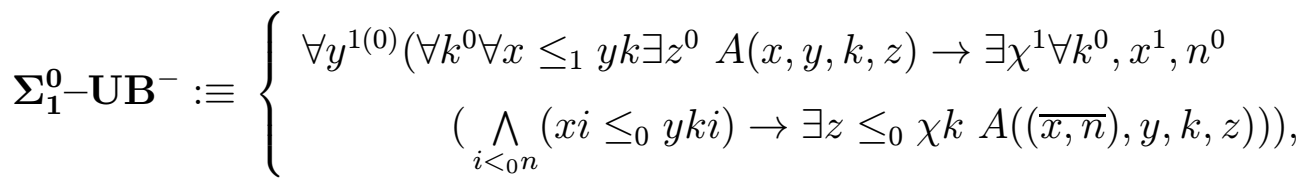

where $A \equiv \exists l^{0} A_{0}(l)$ is a purely existential formula.

$\Sigma_{1}^{0}-\mathrm{UB}^{-}$follows from $F^{-}$relative to $\mathrm{G}_{n} \mathrm{~A}^{\omega}+\mathrm{AC}^{1,0}$-qf (for $n \geq 2$ ).

In $\mathrm{G}_{2} \mathrm{~A}^{\omega}+\Sigma_{1}^{0}-\mathrm{UB}^{-}$and hence in $\mathrm{G}_{2} \mathrm{~A}^{\omega}+F^{-}+\mathrm{AC}^{1,0}{ }_{-\mathrm{qf}}$ one can give very short and perspicuous proofs of various important analytical theorems like

- Every pointwise continuous function $f:[0,1]^{d} \rightarrow \mathbb{R}$ is uniformly continuous

- The attainment of the maximum value of $f \in C\left([0,1]^{d}, \mathbb{R}\right)$ on $[0,1]^{d}$

- The sequential form of the Heine-Borel covering property for $[0,1]^{d}$

- Dini's theorem

- The existence of a uniformly continuous inverse function for every strictly increasing continuous function $f:[0,1] \rightarrow \mathbb{R}$. 
Since $F^{-}$does not contribute to the growth of extractable bounds one can extract polynomial bounds from proofs in $\mathrm{G}_{2} \mathrm{~A}^{\omega}+\Sigma_{1}^{0}-\mathrm{UB}^{-}+\mathrm{AC}-\mathrm{qf}$.

Whereas the straightforward generalization of $\Sigma_{1}^{0}-\mathrm{UB}^{-}$to $\Pi_{k}^{0}$-formulas is not consistent with $\mathrm{G}_{n} \mathrm{~A}^{\omega}$ (see [15]), the following restricted form is (although it does - like $\Sigma_{1}^{0} \mathrm{UB}^{-}$- not hold in the full set-theoretic type structure):

Definition 5.1 Let $\rho=0(0)(0)(1(0))(1), k \geq 0$.

$\Pi_{k}^{0}-U B^{-} \backslash(g): \equiv\left\{\begin{array}{l}\forall \Phi^{\rho}, y^{1(0)}, a^{0}\left(\forall k^{0} \forall x \leq_{1} y k \exists z^{0} A(g, \Phi(x, y, k, z), k, z, a) \rightarrow\right. \\ \left.\exists \chi^{1} \forall k^{0} \forall x \leq_{1} y k \forall l^{0} \exists z \leq_{0} \chi k A(g, \Phi((\overline{x, l}), y, k, z), k, z, a)\right),\end{array}\right.$

where $A\left(g, v^{0}, k^{0}, z^{0}, a^{0}\right): \equiv \forall u_{1}^{0} \exists u_{2}^{0} \ldots \exists^{(d)} u_{k}^{0}\left(g(v, k, z, a, \underline{u})={ }_{0} 0\right) \in \Pi_{k}^{0}$.

Remark 5.2 $G_{n} A^{\omega} \vdash \Pi_{0}^{0}-U B^{-} \uparrow(t) \rightarrow \Sigma_{1}^{0}$-UB $B^{-}$, where $t \in G_{1} R^{\omega}$ such that $t(v, k, z, a)={ }_{0} v$.

In [15] we have shown that every single (sequence of) instance(s) of the Bolzano-Weierstraß principle for bounded sequences in $\mathbb{R}^{d}$ and of the Ascolilemma (in the sense of [22]) follows from suitable instances of $\Pi_{1}^{0}-\mathrm{UB}^{-} \uparrow$ and used this to calibrate precisely the contribution of such instances to the growth of extractable bounds. This indicates the mathematical relevance of our generalized principles of uniform boundedness.

Proposition 5.3 Let $n \geq 2, k \geq 0$. For suitable $\xi \in G_{n} R^{\omega}$ we have

$$
G_{n} A^{\omega}+A C^{1,0}-q f \vdash F^{-}+\Pi_{k}^{0}-C A(\xi g) \rightarrow \Pi_{k}^{0}-U B^{-} \uparrow(g),
$$

where $g$ is a free (function) variable.

\section{Proof:}

For a suitable $\xi \in \mathrm{G}_{2} \mathrm{R}^{\omega}, \Pi_{k}^{0}$-CA $(\xi g)$ yields the existence of a function $h$ such that

$$
\forall v^{0}, k^{0}, z^{0}, a^{0}\left(h v k z a={ }_{0} 0 \leftrightarrow A(g, v, k, z, a)\right),
$$

where $A$ is as in definition 5.1. Using $h$, the assumption of $\Pi_{k}^{0}-\mathrm{UB}^{-} \uparrow(g)$ can be expressed as

$$
\forall k^{0} \forall x \leq_{1} y k \exists z^{0}\left(h(\Phi(x, y, k, z), k, z, a)={ }_{0} 0\right) .
$$

By $\Sigma_{1}^{0}$-UB ${ }^{-}$, which follows from $F^{-}$and $\mathrm{AC}^{1,0}$-qf relative to $\mathrm{G}_{n} \mathrm{~A}^{\omega}$ (see [12]), this yields

$$
\exists \chi^{1} \forall k^{0} \forall x \leq_{1} y k \forall l^{0} \exists z \leq_{0} \chi k\left(h(\Phi((\overline{x, l}), y, k, z), k, z, a)={ }_{0} 0\right)
$$


and hence

$$
\exists \chi^{1} \forall k^{0} \forall x \leq_{1} y k \forall l^{0} \exists z \leq_{0} \chi k A(g, \Phi((\overline{x, l}), y, k, z), k, z, a) .
$$

Using proposition 5.3 we can strengthen proposition 4.3 and corollary 4.4 to

Theorem 5.4 Let $n \geq 3, k \geq 0$ and

$B: \equiv \forall u^{1} \forall v \leq_{\tau} t u \exists a_{1}^{0} \forall b_{1}^{0} \ldots \exists a_{l}^{0} \forall b_{l}^{0} \exists w^{\gamma} B_{0}$ be a sentence in $\mathcal{L}\left(G_{n} A^{\omega}\right)$, where $B_{0}$ is quantifier-free and $t \in G_{n} R^{\omega}$. Let $\xi_{1}, \xi_{2}, \xi_{3} \in G_{n} R^{\omega}$ (of suitable types) and $\Delta$ a set of sentences having the form $\forall x^{\delta} \exists y \leq_{\rho} s x \forall z^{\eta} A_{0}$ ( $A_{0}$ quantifierfree, $\left.s \in G_{n} R^{\omega}\right)$. Then for a suitable $\xi \in G_{n} R^{\omega}$ the following holds:

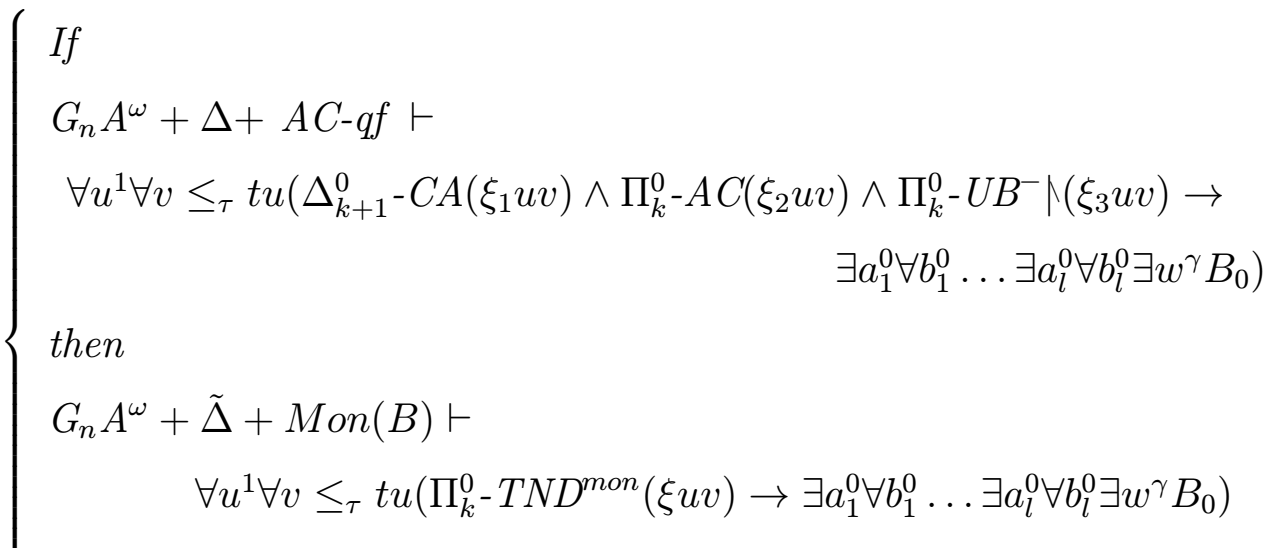

and in particular

$G_{n} A^{\omega}+\Sigma_{k}^{0}-I A+\tilde{\Delta}+M o n(B) \vdash \forall u^{1} \forall v \leq_{\tau} t u \exists a_{1}^{0} \forall b_{1}^{0} \ldots \exists a_{l}^{0} \forall b_{l}^{0} \exists w^{\gamma} B_{0}$.

In the assumption of the rule the theory $G_{n} A^{\omega}+\Delta+A C$-qf can be strengthened to $\left(G_{n} A^{\omega}+\Delta+A C-q f\right) \oplus F^{-}$.

Corollary 5.5 Let $k \geq 1, \gamma \leq 2$ and $\xi_{1}, \xi_{2}, \xi_{3} \in G_{n} R^{\omega}$. Then the following rule holds

$$
\left\{\begin{array}{l}
G_{\infty} A^{\omega}+\Delta+A C-q f \vdash \forall u^{1} \forall v \leq_{\tau} t u \\
\quad\left(\Delta_{k+1}^{0}-C A\left(\xi_{1} u v\right) \wedge \Pi_{k}^{0}-A C\left(\xi_{2} u v\right) \wedge \Pi_{k}^{0}-U B^{-} \wedge\left(\xi_{3} u v\right) \rightarrow \exists w^{\gamma} B_{0}(u, v, w)\right) \\
\Rightarrow \exists \Phi \in T_{k-1} \text { such that } \\
\mathrm{PA}_{i}^{\omega}+\tilde{\Delta} \vdash \forall u^{1} \forall v \leq_{\tau} t u \exists w \leq_{\gamma} \Phi u B_{0}(u, v, w) .
\end{array}\right.
$$

Again we may strengthen the theory in the assumption of the rule above by $\oplus F^{-}$. 
We now show that $\Pi_{k}^{0}-\mathrm{CA}(f)$ in fact is implied by suitable instances of $\Pi_{k}^{0}-\mathrm{UB}^{-} \uparrow$ :

Proposition 5.6 Let $n \geq 2, k \geq 1$. For suitable $\xi_{1}, \ldots, \xi_{l} \in G_{2} R^{\omega}$ we have

$$
G_{n} A^{\omega} \vdash \bigwedge_{i=1}^{l} \Pi_{k}^{0}-U B^{-} \uparrow\left(\xi_{i} f\right) \rightarrow \Pi_{k}^{0}-C A(f)
$$

where $f$ is a free (function) variable.

Proof: Induction on $k . k=1: \Pi_{1}^{0}-\mathrm{CA}(f)$ is logically equivalent to

(1) $\exists g \leq_{1} 1 \forall x^{0}, y^{0} \exists z^{0}\left(\left(g x={ }_{0} 0 \rightarrow f(x, y)={ }_{0} 0\right) \wedge\left(f(x, z)={ }_{0} 0 \rightarrow g x={ }_{0} 0\right)\right)$

and hence to $(2): \equiv$

$\neg \forall g \leq{ }_{1} 1 \exists x^{0}, y^{0} \forall z^{0} \neg\left(\left(g x={ }_{0} 0 \rightarrow f(x, y)={ }_{0} 0\right) \wedge\left(f(x, z)={ }_{0} 0 \rightarrow g x={ }_{0} 0\right)\right)$.

For a suitable $\xi_{1} \in \mathrm{G}_{2} \mathrm{R}^{\omega}, \Pi_{1}^{0}$-UB $\mathrm{UB}^{-} \uparrow\left(\xi_{1} f\right)$ yields the equivalence of (2) and

$$
\left\{\begin{aligned}
\neg \exists n^{0} \forall g & \leq_{1} 1 \exists x, y \leq n \forall z^{0} \\
& \neg\left(\left(g x={ }_{0} 0 \rightarrow f(x, y)={ }_{0} 0\right) \wedge\left(f(x, z)={ }_{0} 0 \rightarrow g x={ }_{0} 0\right)\right)
\end{aligned}\right.
$$

i.e.

$$
\left\{\begin{array}{l}
\forall n^{0} \exists g \leq_{1} 1 \forall x \leq n \\
\quad\left(\left(g x={ }_{0} 0 \rightarrow \forall y \leq n f(x, y)={ }_{0} 0\right) \wedge\left(\forall z\left(f(x, z)={ }_{0} 0\right) \rightarrow g x={ }_{0} 0\right)\right)
\end{array}\right.
$$

Define

$$
g x:=\left\{\begin{array}{l}
0^{0} \text { if } \forall y \leq n(f(x, y)=0) \\
1^{0} \text { otherwise. }
\end{array}\right.
$$

Let $k \geq 1 . k \mapsto k+1$ :

$\Pi_{k+1}^{0}-\mathrm{CA}(f)$ is equivalent to

$$
(*)\left\{\begin{array}{c}
\exists g \leq_{1} 1 \forall x^{0}, y^{0} \exists z^{0}\left(\left(g x={ }_{0} 0 \rightarrow \exists u_{1}^{0} \forall u_{2}^{0} \ldots \forall^{(d)} u_{k}^{0}\left(f(x, y, \underline{u})={ }_{0} 0\right)\right) \wedge\right. \\
\left.\left(\exists u_{1}^{0} \forall u_{2}^{0} \ldots \forall^{(d)} u_{k}^{0}\left(f(x, z, \underline{u})={ }_{0} 0\right) \rightarrow g x=0\right)\right) .
\end{array}\right.
$$

By induction hypothesis there exists an instance $\Pi_{k}^{0}-\mathrm{UB}^{-} \uparrow\left(\xi_{2} f\right)$ (which can be considered as an instance $\Pi_{k+1}^{0}-\mathrm{UB}^{-} \uparrow\left(\xi_{2} f\right)$ ) which implies (relative to $\mathrm{G}_{n} \mathrm{~A}^{\omega}$ ) $\Pi_{k}^{0}-\mathrm{CA}(f)$ and hence the existence of an $h$ such that

$$
\forall x, a\left(h(x, a)={ }_{0} 0 \leftrightarrow \exists u_{1} \forall u_{2} \ldots \forall^{(d)} u_{k}\left(f(x, a, \underline{u})={ }_{0} 0\right)\right) .
$$


By $\Pi_{k+1}^{0}-\mathrm{UB}^{-} \uparrow\left(\xi_{3} f\right)$ (for a suitable $\left.\xi_{3}\right)$ applied to the negation of $(*)$, $\Pi_{k+1}^{0}-\mathrm{CA}(f)$ is equivalent to

$$
(* *)\left\{\begin{array}{r}
\forall n \exists g \leq_{1} 1 \forall x \leq n\left(\left(g x={ }_{0} 0 \rightarrow \forall y \leq n \exists u_{1}^{0} \forall u_{2}^{0} \ldots \forall^{(d)} u_{k}^{0} f(x, y, \underline{u})={ }_{0} 0\right)\right. \\
\left.\wedge\left(\forall z \exists u_{1}^{0} \forall u_{2}^{0} \ldots \forall^{(d)} u_{k}^{0} f(x, z, \underline{u})=0 \rightarrow g x={ }_{0} 0\right)\right),
\end{array}\right.
$$

which is satisfied by

$$
g x:=\left\{\begin{array}{l}
0^{0} \text { if } \forall y \leq n(h(x, y)=0) \\
1^{0} \text { otherwise }
\end{array}\right.
$$

Corollary 5.7 For $n \geq 2, k \geq 1$ the following holds:

1) $G_{n} A^{\omega} \vdash \forall g \Pi_{1}^{0}-U B^{-} \uparrow(g) \rightarrow \forall \tilde{g} \Pi_{k}^{0}-C A(\tilde{g})$.

2) $G_{n} A^{\omega} \vdash \forall g \Pi_{1}^{0}-U B^{-} \uparrow(g) \leftrightarrow \forall \tilde{g} \Pi_{k}^{0}-U B^{-} \uparrow(\tilde{g})$.

Proof: 1) By proposition $5.6 \forall g \Pi_{1}^{0}$-UB $^{-} \uparrow(g)$ implies $\forall f \Pi_{1}^{0}-\mathrm{CA}(f)$ and hence $\forall f \Pi_{k}^{0}-\mathrm{CA}(f)$ (by iteration).

2) follows from 1) and the proof of proposition 5.3.

Let $\mathrm{B}_{0,1}$ be the type-0-bar recursor constant of equality rank 1 , i.e. $\mathrm{B}_{0,1}$ is characterized by the axioms

$$
\left(\mathrm{BR}_{0,1}\right):\left\{\begin{array}{l}
x^{2}\left(\overline{y^{1}, n^{0}}\right)<n \rightarrow \mathrm{B}_{0,1} \text { xzuny }={ }_{1} z \\
x(\overline{y, n}) \geq n \rightarrow \mathrm{B}_{0,1} x z \text { uny }=_{1} u\left(\lambda D^{0} . \mathrm{B}_{0,1} x z u n^{\prime}(\overline{y, n} * D)\right)
\end{array}\right.
$$

where $u$ is of type $1(1(0))$ and

$$
(\overline{y, n} * D)\left(k^{0}\right)={ }_{1}\left\{\begin{array}{l}
x k, \text { if } k<n \\
D, \text { if } k=n \\
0^{1}, \text { otherwise }
\end{array}\right.
$$

Proposition 5.8 Let $n \geq 3, k \geq 1, B_{0}(u, v, w)$ be a quantifer-free formula of $G_{n} A^{\omega}$ containing only $u, v, w$ free, $t^{\tau 1} \in G_{n} R^{\omega}, \gamma \leq 2$. Then the following rule holds:

$$
\left\{\begin{array}{l}
G_{n} A^{\omega}+\Delta+A C-q f \vdash \forall g \Pi_{k}^{0}-U B^{-} \wedge(g) \rightarrow \forall u^{1} \forall v \leq_{\tau} t u \exists w^{\gamma} B_{0}(u, v, w) \\
\Rightarrow \exists \Phi \in G_{n} R^{\omega}\left[\mathrm{B}_{0,1}\right] \text { such that } \\
G_{n} A^{\omega}+\tilde{\Delta}+\left(\mathrm{BR}_{0,1}\right)+\left(D C^{0}\right) \vdash \forall u^{1} \forall v \leq_{\tau} t u \exists w \leq_{\gamma} \Phi u B_{0}(u, v, w),
\end{array}\right.
$$


where

$$
\left(D C^{0}\right): \equiv \forall x^{0} \exists y^{0} A(x, y) \rightarrow \forall x^{0} \exists z^{1}\left(z 0={ }_{0} x \wedge \forall z_{1}^{0} A\left(z z_{1}, z\left(z_{1}^{\prime}\right)\right)\right) .
$$

Proof: By proposition 5.3 and corollary 5.7 one has

$$
\mathrm{G}_{n} \mathrm{~A}^{\omega}+\mathrm{AC}^{1,0}{ }_{-\mathrm{qf}}+\forall g \Pi_{1}^{0}-\mathrm{CA}(g) \vdash F^{-} \rightarrow \forall \tilde{g} \Pi_{k}^{0}-\mathrm{UB}^{-} \uparrow(\tilde{g}) .
$$

Hence the assumption of the rule to be proved yields

$$
\mathrm{G}_{n} \mathrm{~A}^{\omega}+\Delta+\mathrm{AC}-\mathrm{qf}+\forall g \Pi_{1}^{0}-\mathrm{CA}(g) \vdash F^{-} \rightarrow \forall u^{1} \forall v \leq_{\tau} t u \exists w^{\gamma} B_{0}(u, v, w) .
$$

From the work of Spector [23] it follows that $\mathrm{G}_{n} \mathrm{~A}^{\omega}+\mathrm{AC}-\mathrm{qf}+\forall g \Pi_{1}^{0}-\mathrm{CA}(g)$ has (via negative translation) a Gödel functional interpretation in $\mathrm{G}_{n} \mathrm{~A}_{i}^{\omega}+$ $\left(\mathrm{BR}_{0,1}\right)$ by terms $\in \mathrm{G}_{n} \mathrm{R}^{\omega}\left[\mathrm{B}_{0,1}\right]$. In [2] it is shown that the type structure $\mathcal{M}^{\omega}$ of the so-called strongly majorizable functionals forms a model of full bar recursion. From the proof of this fact (restricted to type-0-bar recursion) one obtains the construction of a term $\mathrm{B}_{0,1}^{*} \in \mathrm{G}_{n} \mathrm{R}^{\omega}\left[\mathrm{B}_{0,1}\right]$ such that

$$
\mathrm{G}_{n} \mathrm{~A}^{\omega}+\left(\mathrm{BR}_{0,1}\right)+\left(D C^{0}\right) \vdash \mathrm{B}_{0,1}^{*} \mathrm{~s}-\mathrm{maj} \mathrm{B}_{0,1}
$$

where 's-maj' is the corresponding syntactic notion of strong majorization as definined in definition 2.1. Therefore the proof of the fact that (the negative translation of) $\mathrm{G}_{n} \mathrm{~A}^{\omega}+\mathrm{AC}-\mathrm{qf}+\Delta$ has a monotone functional interpretation (in the sense of [9]) in $\mathrm{G}_{n} \mathrm{~A}_{i}^{\omega}$ by terms in $\mathrm{G}_{n} \mathrm{R}^{\omega}$ (see [12]) extends to $\mathrm{G}_{n} \mathrm{~A}^{\omega}+\Delta+$ AC-qf $+\forall g \Pi_{1}^{0}-\mathrm{CA}(g)$ yielding a monotone functional interpretation (via negative translation) in $\mathrm{G}_{n} \mathrm{~A}^{\omega}+\tilde{\Delta}+\left(\mathrm{BR}_{0,1}\right)+\left(D C^{0}\right)$ by terms in $\mathrm{G}_{n} \mathrm{R}^{\omega}\left[\mathrm{B}_{0,1}\right]$. This has the consequence that as in the case of $\mathrm{G}_{n} \mathrm{~A}^{\omega}+\Delta+$ AC-qf (see the proof of theorem 4.21 in [12]) we can eliminate $F^{-}$from the proof of $\forall u \forall v \leq t u \exists w B_{0}$ and extract a uniform bound $\Phi$ on ' $\exists w$ ' which now of course is only in $\mathrm{G}_{n} \mathrm{R}^{\omega}\left[\mathrm{B}_{0,1}\right]$ (instead of $\mathrm{G}_{n} \mathrm{R}^{\omega}$ ) and its verification can be carried out in $\mathrm{G}_{n} \mathrm{~A}^{\omega}+\tilde{\Delta}+\left(\mathrm{BR}_{0,1}\right)+\left(D C^{0}\right)$.

\section{References}

[1] Beklemishev, L.D., A proof-theoretic analysis of collection. Preprint 1996.

[2] Bezem, M.A., Strongly majorizable functionals of finite type: a model for bar recursion containing discontinuous functionals. J. Symb. Logic 50 pp. 652-660 (1985). 
[3] Clote, P., Hájek, P., Paris, J., On some formalized conservation results in arithmetic. Arch. Math. Logic 30, pp. 201-218 (1990).

[4] Gödel, K., Zur intuitionistischen Arithmetik und Zahlentheorie. Ergebnisse eines Mathematischen Kolloquiums, vol. 4 pp. 34-38 (1933).

[5] Gödel, K., Über eine bisher noch nicht benutzte Erweiterung des finiten Standpunktes. Dialectica 12, pp. 280-287 (1958).

[6] Howard, W.A., Hereditarily majorizable functionals of finite type. In: Troelstra (1973).

[7] Kay, R., Models of Peano Arithmetic. Oxford Logic Guides 15, Clarendon Press Oxford 1991.

[8] Kohlenbach, U., Pointwise hereditary majorization and some applications. Arch. Math. Logic 31, pp.227-241 (1992).

[9] Kohlenbach, U., Analysing proofs in analysis. In: W. Hodges, M. Hyland, C. Steinhorn, J. Truss, editors, Logic: from Foundations to Applications. European Logic Colloquium (Keele, 1993), pp. 225-260, Oxford University Press (1996).

[10] Kohlenbach, U., Real growth in standard parts of analysis. Habilitationsschrift, pp. xv+166, Frankfurt (1995).

[11] Kohlenbach, U., A note on the $\Pi_{2}^{0}$-induction rule. Arch. Math. Logic 34, pp. 279-283 (1995).

[12] Kohlenbach, U., Mathematically strong subsystems of analysis with low rate of provably recursive functionals. Arch. Math. Logic 36, pp. 31-71 (1996).

[13] Kohlenbach, U., Elimination of Skolem functions for monotone formulas. To appear in: Archive for Mathematical Logic.

[14] Kohlenbach, U., The use of a logical principle of uniform boundedness in analysis. To appear in: Proc. 'Logic in Florence 1995'.

[15] Kohlenbach, U., Arithmetizing proofs in analysis. To appear in: Proc. Logic Colloquium '96 (San Sebastian).

[16] Luckhardt, H., Extensional Gödel functional interpretation. A consistency proof of classical analysis. Springer Lecture Notes in Mathematics 306 (1973). 
[17] Paris, J., Kirby, L., $\Sigma_{n}^{0}$-collection schema in arithmetic. In: Logic Colloquium '77, pp. 199-209. North-Holland, Amsterdam, 1978.

[18] Parsons, C., On a number theoretic choice schema and its relation to induction. In: Intuitionism and proof theory, pp. 459-473. North-Holland, Amsterdam (1970).

[19] Parsons, C., On n-quantifier induction. J. Symbolic Logic 37, pp. 466482 (1972).

[20] Shoenfield, J.R., Mathematical Logic, Addison-Wesley, New York, 1967.

[21] Sieg, W., Fragments of arithmetic. Ann. Pure Appl. Logic 28, pp. 33-71 (1985).

[22] Simpson, S.G., Which set existence axioms are needed to prove the Cauchy/Peano theorem for ordinary differential equations. J. Symbolic Logic 49,pp. 783-801 (1984).

[23] Spector, C., Provably recursive functionals of analysis: a consistency proof of analysis by an extension of principles formulated in current intuitionistic mathematics. In: Recursive function theory, Proceedings of Symposia in Pure Mathematics, vol. 5 (J.C.E. Dekker (ed.)), AMS, Providence, R.I., pp. 1-27 (1962).

[24] Troelstra, A.S. (ed.) Metamathematical investigation of intuitionistic arithmetic and analysis. Springer Lecture Notes in Mathematics 344 (1973). 


\section{Recent BRICS Report Series Publications}

RS-97-23 Ulrich Kohlenbach. On the Arithmetical Content of Restricted Forms of Comprehension, Choice and General Uniform Boundedness. August 1997. 35 pp.

RS-97-22 Carsten Butz. Syntax and Semantics of the logic $\mathcal{L}_{\omega \omega}^{\lambda}$. July 1997. 14 pp.

RS-97-21 Steve Awodey and Carsten Butz. Topological Completeness for Higher-Order Logic. July 1997. 19 pp.

RS-97-20 Carsten Butz and Peter T. Johnstone. Classifying Toposes for First Order Theories. July 1997. 34 pp.

RS-97-19 Andrew D. Gordon, Paul D. Hankin, and Søren B. Lassen. Compilation and Equivalence of Imperative Objects. July 1997. iv+64 pp. Aappears also as Technical Report 429, University of Cambridge Computer Laboratory, June 1997. To appear in Foundations of Software Technology and Theoretical Computer Science: 17th Conference, FCT\&TCS '97 Proceedings, LNCS, 1997.

RS-97-18 Robert Pollack. How to Believe a Machine-Checked Proof. July 1997. 18 pp. To appear as a chapter in the book Twenty Five Years of Constructive Type Theory, eds. Smith and Sambin, Oxford University Press.

RS-97-17 Peter Bro Miltersen. Error Correcting Codes, Perfect Hashing Circuits, and Deterministic Dynamic Dictionaries. June 1997. 10 pp.

RS-97-16 Noga Alon, Martin Dietzfelbinger, Peter Bro Miltersen, Erez Petrank, and Gábor Tardos. Linear Hashing. June 1997. 22 pp. A preliminary version appeared with the title Is Linear Hashing Good? in The Twenty-ninth Annual ACM Symposium on Theory of Computing, STOC '97, pages 465-474.

RS-97-15 Pierre-Louis Curien, Gordon Plotkin, and Glynn Winskel. Bistructures, Bidomains and Linear Logic. June 1997. 41 pp.

RS-97-14 Arne Andersson, Peter Bro Miltersen, Søren Riis, and Mikkel Thorup. Dictionaries on $A C^{0} R A M s:$ Query Time $\Theta(\sqrt{\log n / \log \log n})$ is Necessary and Sufficient. June 1997. 18 pp. Appears in 37th Annual Symposium on Foundations of Computer Science, FOCS '96 Proceedings, pages 441-450. 